\title{
Natural NMSSM after LHC Run I and the Higgsino dominated dark matter scenario
}

\author{
Junjie Cao, ${ }^{a, b}$ Yangle $\mathrm{He},{ }^{a}$ Liangliang Shang, ${ }^{a}$ Wei Su ${ }^{c, d}$ and Yang Zhang ${ }^{c, d}$ \\ ${ }^{a}$ Department of Physics, Henan Normal University, \\ Jianshe East Road, Xinxiang 453007, China \\ ${ }^{b}$ Department of Applied Physics, Xi'an Jiaotong University, \\ Xianning West Road, Xi'an 710049, China \\ ${ }^{c}$ CAS Key Laboratory of Theoretical Physics, \\ Institute of Theoretical Physics, Chinese Academy of Sciences, \\ Zhong Guan Cun East Street, Beijing 100190, China \\ ${ }^{d}$ School of Physical Sciences, University of Chinese Academy, \\ Yuquan Road, Beijing 100049, China \\ E-mail: junjiec@itp.ac.cn, heyangle90@gmail.com, \\ shlwell1988@gmail.com, weisv@itp.ac.cn, zhangyang@itp.ac.cn
}

ABSTRACT: We investigate the impact of the direct searches for SUSY at LHC Run I on the naturalness of the Next-to-Minimal Supersymmetric Standard Model (NMSSM). For this end, we first scan the vast parameter space of the NMSSM to get the region where the fine tuning measures $\Delta_{Z}$ and $\Delta_{h}$ at the electroweak scale are less than about 50, then we implement by simulations the constraints of the direct searches on the parameter points in the region. Our results indicate that although the direct search experiments are effective in excluding the points, the parameter intervals for the region and also the minimum reaches of $\Delta_{Z}$ and $\Delta_{h}$ are scarcely changed by the constraints, which implies that the fine tuning of the NMSSM does not get worse after LHC Run I. Moreover, based on the results we propose a natural NMSSM scenario where the lightest neutralino $\tilde{\chi}_{1}^{0}$ as the dark matter (DM) candidate is Higgsino-dominated. In this scenario, $\Delta_{Z}$ and $\Delta_{h}$ may be as low as 2 without conflicting with any experimental constraints, and intriguingly $\tilde{\chi}_{1}^{0}$ can easily reach the measured DM relic density due to its significant Singlino component. We exhibit the features of the scenario which distinguish it from the other natural SUSY scenario, including the properties of its neutralino-chargino sector and scalar top quark sector. We emphasize that the scenario can be tested either through searching for $3 l+E_{T}^{\text {miss }}$ signal at $14 \mathrm{TeV}$ LHC or through future DM direct detection experiments.

KEYWORDS: Supersymmetry Phenomenology

ARXIV EPrint: 1606.04416 


\section{Contents}

1 Introduction 1

2 The structure of the NMSSM and our scan strategy 4

2.1 The structure of the NMSSM 4

$\begin{array}{lll}2.2 & \text { Strategy in scanning the parameter space of the NMSSM } & 7\end{array}$

3 Key features of the NS scenario with $\tilde{\chi}_{1}^{0}$ being Higgsino-dominated 11

4 Future detection of our scenario $\quad 13$

$\begin{array}{lll}4.1 & \text { Detection at } 14 \mathrm{TeV} \text { LHC } & 13\end{array}$

$\begin{array}{lll}4.2 & \text { Dark matter direct search } & 15\end{array}$

5 Conclusions $\quad 16$

A Fastlim and SModelS $\quad 18$

$\begin{array}{llr}\text { A.1 Fastlim } & 18\end{array}$

$\begin{array}{llr}\text { A.2 SModelS } & 18\end{array}$

$\begin{array}{ll}\text { B Details of our simulation } & 19\end{array}$

$\begin{array}{lll}\text { B.1 Search for electroweakino at the LHC } & 19\end{array}$

B.2 Search for stops at the LHC 20

\section{Introduction}

In the supersymmetric models such as the Minimal Supersymmetric Standard Model (MSSM) $[1,2]$ and the Next-to-Minimal Supersymmetric Standard Model (NMSSM) [3, 4], the $Z$ boson mass is given by [5]

$$
m_{Z}^{2}=\frac{2\left(m_{H_{d}}^{2}+\Sigma_{d}\right)-2\left(m_{H_{u}}^{2}+\Sigma_{u}\right) \tan ^{2} \beta}{\tan ^{2} \beta-1}-2 \mu^{2},
$$

where $m_{H_{d}}^{2}$ and $m_{H_{u}}^{2}$ represent the weak scale soft SUSY breaking masses of the Higgs fields $H_{d}$ and $H_{u}$ respectively, $\Sigma_{d}$ and $\Sigma_{u}$ are their radiative corrections, $\mu$ is the Higgsino mass and $\tan \beta \equiv v_{u} / v_{d}$. As was shown in [6], the corrections $\Sigma_{d}$ and $\Sigma_{u}$ can be obtained from the effective Higgs potential at loop level, and in case of a large $\tan \beta$, their largest contributions arise from the Yukawa interactions of third generation squarks, which are

$$
\begin{aligned}
& \Sigma_{u} \simeq \sum_{i=1}^{2} \frac{3 Y_{t}^{2}}{16 \pi^{2}} \times m_{\tilde{t}_{i}}^{2}\left(\log \frac{m_{\tilde{t}_{i}}^{2}}{Q^{2}}-1\right), \\
& \Sigma_{d} \simeq \sum_{i=1}^{2} \frac{3 Y_{b}^{2}}{16 \pi^{2}} \times m_{\tilde{b}_{i}}^{2}\left(\log \frac{m_{\tilde{b}_{i}}^{2}}{Q^{2}}-1\right) .
\end{aligned}
$$


In above formulae, $Q$ denotes the renormalization scale in getting the effective potential, and its optimized value is usually taken as $Q=\sqrt{m_{\tilde{t}_{1}} m_{\tilde{t}_{2}}}$ with $\tilde{t}_{1}$ and $\tilde{t}_{2}$ being the light and heavy top squarks (stop) respectively. Obviously, if the observed value of $m_{Z}$ is obtained without resorting to large cancelations, each term on the right hand side of eq. (1.1) should be comparable in magnitude with $m_{Z}^{2}$, and this in return can put non-trivial constraints on the magnitudes of $\mu$ and $m_{\tilde{t}_{1,2}}$. Numerically speaking, we find that requiring the individual term to be less than $10 m_{Z}^{2}$ leads to $\mu$ and $m_{\tilde{t}_{1,2}}$ upper bounded by about $200 \mathrm{GeV}$ and $1.5 \mathrm{TeV}$ respectively. In history, the scenario satisfying the bounds is dubbed as Natural SUSY (NS) [5].

In the MSSM, the NS scenario is theoretically unsatisfactory due to at least three considerations. First, since the Higgsino mass $\mu$ is the only dimensionful parameter in the superpotential of the MSSM, its typical size should be of the order of the SUSY breaking scale. Given that the LHC searches for supersymmetric particles have pushed the masses of gluinos and first generation squarks up to above $1 \mathrm{TeV}[7,8], \mu \lesssim 200 \mathrm{GeV}$ seems rather unnatural. Second, the relic density of the dark matter (DM) predicted in the NS scenario is hardly to coincide with its measured value. Explicitly speaking, it has been shown that if the DM $\tilde{\chi}_{1}^{0}$ is Higgsino-dominated, ${ }^{1}$ its density is usually about one order smaller than its measured value [5], alternatively if it is Bino-dominated, the correct density can be achieved only in very limited parameter regions of the MSSM [9]. These features make the NS scenario disfavored by DM physics. Third, the NS scenario is further exacerbated by the uncomfortably large mass of the recently discovered Higgs particle [10, 11]: its value $m_{h} \simeq 125 \mathrm{GeV}$ lies well beyond its tree-level upper bound $m_{h} \leq m_{Z}$, and consequently stops heavier than about $1 \mathrm{TeV}$ must be present to provide a large radiative correction to the mass [12-17]. This requirement seems in tension with the naturalness argument of eq. (1.1). In fact, all these problems point to the direction that the NS scenario should be embedded in a more complex framework. Remarkably, we note that the NMSSM is an ideal model to alleviate these problems.

The NMSSM extends the MSSM by one gauge singlet superfield $\hat{S}$, and it is the simplest SUSY extension of the Standard Model (SM) with a scale invariant superpotential (i.e. its superpotential does not contain any dimensionful parameters) [3, 4]. In this model, the Higgsino mass $\mu$ is dynamically generated by the vacuum expectation value of $\hat{S}$, and given that all singlet-dominated scalars are lighter than about $v \simeq 174 \mathrm{GeV}$, its magnitude can be naturally less than $200 \mathrm{GeV}$. These additional singlet-dominated scalars, on the other hand, can act as the mediator or final states of the DM annihilation [18], and consequently the NS scenario in the NMSSM with a Singlino-dominated DM can not only predict the correct relic density, but also explain the galactic center $\gamma$-ray excess [18-21]. Moreover, in the NMSSM the interaction $\lambda \hat{S} \hat{H}_{u} \cdot \hat{H}_{d}$ can lead to a positive contribution to the squared mass of the SM-like Higgs boson, and if the boson corresponds to the next-to-lightest CP-even Higgs state, its mass can be further lifted up by the singlet-doublet Higgs mixing. These

\footnotetext{
${ }^{1}$ Throughout this work, we denote the mass eigenstates of the neutralinos by $\tilde{\chi}_{i}^{0}$ with $i$ ranging from 1 to 4 (5) for MSSM (NMSSM), and assume an ascending mass order for the $\tilde{\chi}_{i}^{0}$ by convention. With such an assumption, the lightest neutralino $\tilde{\chi}_{1}^{0}$ as the lightest supersymmetric particle (LSP) is regarded as the DM candidate.
} 
enhancements make the large radiative correction of the stops unnecessary in predicting $m_{h} \simeq 125 \mathrm{GeV}$, and thus stops can be relatively light [22-29].

So far studies on the NS scenario in the NMSSM are concentrated on the assumption that $\tilde{\chi}_{1}^{0}$ is Singlino-dominated [18-21,30-46]. In this case, the branching ratio of the golden channel $\tilde{t}_{1} \rightarrow t \tilde{\chi}_{1}^{0}$ in the LHC search for a moderately light stop is highly suppressed. Instead, $\tilde{t}_{1}$ mainly decays into the Higgsino-dominated $\tilde{\chi}_{1}^{+}$and $\tilde{\chi}_{2,3}^{0}$ in following way [43]

$$
\tilde{t}_{1} \rightarrow b \tilde{\chi}_{1}^{+} \rightarrow b W^{+(*)} \tilde{\chi}_{1}^{0}, \quad \tilde{t}_{1} \rightarrow t \tilde{\chi}_{2,3}^{0} \rightarrow t X^{0(*)} \tilde{\chi}_{1}^{0},
$$

where $X^{0}$ denotes either $Z$ boson or a neutral Higgs boson. These lengthened decay chains can generate softer final particles in comparison with the golden channel, and consequently weaken the LHC bounds in the stop search. This feature is also applied to other sparticle searches, and it has been viewed as an advantage of the NMSSM in circumventing the tight constraints from the LHC searches for SUSY. In this work, we consider another realization of the NS scenario where $\tilde{\chi}_{1}^{0}$ is Higgsino-dominated. In our scenario, the Higgsinos and the Singlino are degenerated in mass at $50 \%$ level, and consequently they mix rather strongly to form mass eigenstates $\tilde{\chi}_{1,2,3}^{0}$ with $\tilde{\chi}_{1,2}^{0}$ and $\tilde{\chi}_{3}^{0}$ being Higgsino-dominated and Singlino-dominated respectively. Since the role of the Singlino component in $\tilde{\chi}_{1}^{0}$ is to decrease the DM annihilation rate, $\tilde{\chi}_{1}^{0}$ may achieve the relic density measured by Planck and WMAP experiments $[47,48]$ without contradicting the DM direct search experiments such as LUX [49, 50]. The phenomenology of our scenario is somewhat similar to that of the popular NS scenario in the MSSM, which was proposed in [5], but our scenario has following advantages

- In the parameter regions allowed by current LHC searches for SUSY, it may have a lower fine tuning in getting the $Z$ boson mass. Meanwhile, it has broad parameter regions to predict the right relic density (see our discussions in section 3 ).

- The mass gaps of $\tilde{\chi}_{1}^{ \pm}$and $\tilde{\chi}_{2}^{0}$ from $\tilde{\chi}_{1}^{0}$ are sizable, e.g. $\Delta_{ \pm} \equiv m_{\tilde{\chi}_{1}^{ \pm}}-m_{\tilde{\chi}_{1}^{0}} \gtrsim 30 \mathrm{GeV}$ and $\Delta_{0} \equiv m_{\tilde{\chi}_{2}^{0}}-m_{\tilde{\chi}_{1}^{0}} \gtrsim 50 \mathrm{GeV}$, and consequently the leptons from the decay chains $\tilde{\chi}_{1}^{ \pm} \rightarrow W^{(*)} \tilde{\chi}_{1}^{0} \rightarrow l \nu \tilde{\chi}_{1}^{0}$ and $\tilde{\chi}_{2}^{0} \rightarrow Z^{(*)} \tilde{\chi}_{1}^{0} \rightarrow l l \tilde{\chi}_{1}^{0}$ are usually energetic. As a result, our scenario can be tested at future LHC experiments by the process $p p \rightarrow \tilde{\chi}_{1}^{ \pm} \tilde{\chi}_{2}^{0} \rightarrow$ $3 l+E_{T}^{\text {miss }}$. By contrast, in the NS scenario of the MSSM the leptons are very soft and hardly detectable due to the small mass splittings: $\Delta_{ \pm}, \Delta_{0} \sim \mathcal{O}(1 \mathrm{GeV})[51]$.

- Since all the light particles in our scenario, i.e. $\tilde{\chi}_{1}^{ \pm}, \tilde{\chi}_{1}^{0}, \tilde{\chi}_{2}^{0}$ and $\tilde{\chi}_{3}^{0}$, have sizable $\tilde{H}_{u}$ component, the main decay modes of $\tilde{t}_{1}$ include

$$
\tilde{t}_{1} \rightarrow b \tilde{\chi}_{1}^{+} \rightarrow b W^{*} \tilde{\chi}_{1}^{0}, \quad \tilde{t}_{1} \rightarrow t \tilde{\chi}_{1}^{0}, \quad \tilde{t}_{1} \rightarrow t \tilde{\chi}_{2,3}^{0} \rightarrow t X^{0 *} \tilde{\chi}_{1}^{0},
$$

and each mode corresponds to different signals. Because the signal of $\tilde{t}_{1}$ pair production is shared by rich final states, the LHC bounds on $m_{\tilde{t}_{1}}$ are usually weakened.

Moreover, we remind that the phenomenology of our scenario is different from that of the NS scenario with a Singlino-dominated DM. This can be seen for example from the decay modes of $\tilde{t}_{1}$, which are presented in eq. (1.2) and eq. (1.3) respectively. We also remind 
that our scenario was scarcely discussed in literatures. In fact, within our knowledge only the work [52] briefly commented that $\tilde{\chi}_{1}^{0}$ may be Higgsino-dominated in the constrained NMSSM.

This work is organized as follows. In section 2, we briefly recapitulate the framework of the NMSSM, then we scan its parameter space by considering various constraints to get the NS scenarios in the NMSSM. Especially, we take great pains to implement the constraints from the LHC searches for SUSY by multiple packages and also by detailed Monte Carlo simulations, like what the work [53] did. After these preparation, we exhibit in section 3 the features of the NS scenario with a Higgsino-dominated DM, including its favored spectrum and the properties of the neutralinos and stops, and subsequently in section 4 we take several benchmark points as examples to show the detection of our scenario in future experiments. Finally, we draw our conclusions in section 5. The details of our treatment on the LHC searches for SUSY are presented in the appendix.

\section{The structure of the NMSSM and our scan strategy}

\subsection{The structure of the NMSSM}

The NMSSM extends the MSSM by adding one gauge singlet superfield $\hat{S}$, and since it aims at solving the $\mu$ problem of the MSSM, a $Z_{3}$ discrete symmetry under which the Higgs superfields $\hat{H}_{u, d}$ and $\hat{S}$ are charged is adopted to avoid the appearance of dimensionful parameters in its superpotential. Consequently, the superpotential of the NMSSM can be written as [3]

$$
W_{\mathrm{NMSSM}}=W_{F}+\lambda \hat{H}_{u} \cdot \hat{H}_{d} \hat{S}+\frac{1}{3} \kappa \hat{S^{3}},
$$

where $W_{F}$ is the superpotential of the MSSM without the $\mu$-term, and the dimensionless parameters $\lambda, \kappa$ describe the interactions among the Higgs superfields.

The Higgs potential of the NMSSM is given by the usual F-term and D-term of the superfields as well as the soft breaking terms, which are given by

$$
V_{\mathrm{NMSSM}}^{\mathrm{soft}}=m_{H_{u}}^{2}\left|H_{u}\right|^{2}+m_{H_{d}}^{2}\left|H_{d}\right|^{2}+m_{S}^{2}|S|^{2}+\left(\lambda A_{\lambda} S H_{u} \cdot H_{d}+\frac{1}{3} \kappa A_{\kappa} S^{3}+\text { h.c. }\right),
$$

with $H_{u}, H_{d}$ and $S$ representing the scalar component fields of $\hat{H}_{u}, \hat{H}_{d}$ and $\hat{S}$ respectively. Considering that the physical implication of the fields $H_{u}$ and $H_{d}$ is less clear, one usually introduces following combinations [3]

$$
H_{1}=\cos \beta H_{u}+\varepsilon \sin \beta H_{d}^{*}, \quad H_{2}=\sin \beta H_{u}-\varepsilon \cos \beta H_{d}^{*}, \quad H_{3}=S,
$$

where $\varepsilon$ is second-order antisymmetric tensor with $\varepsilon_{12}=-\varepsilon_{21}=1$ and $\varepsilon_{11}=\varepsilon_{22}=0$, and $\tan \beta \equiv v_{u} / v_{d}$ with $v_{u}$ and $v_{d}$ denoting the vacuum expectation values of $H_{u}$ and $H_{d}$ respectively. In this representation, the redefined fields $H_{i}(i=1,2,3)$ are given by

$$
H_{1}=\left(\begin{array}{c}
H^{+} \\
\frac{S_{1}+i P_{1}}{\sqrt{2}}
\end{array}\right), \quad H_{2}=\left(\begin{array}{c}
G^{+} \\
v+\frac{S_{2}+i G^{0}}{\sqrt{2}}
\end{array}\right), \quad H_{3}=v_{s}+\frac{1}{\sqrt{2}}\left(S_{3}+i P_{2}\right) .
$$


These expressions indicate that the field $H_{2}$ corresponds to the SM Higgs doublet with $G^{+}$ and $G^{0}$ denoting the Goldston bosons eaten by $W$ and $Z$ bosons respectively, and the field $H_{1}$ represents a new $\mathrm{SU}(2)_{L}$ doublet scalar field which has no tree-level couplings to the $\mathrm{W} / \mathrm{Z}$ bosons. These expressions also indicate that the Higgs sector of the NMSSM includes three CP-even mass eigenstates $h_{1}, h_{2}$ and $h_{3}$ which are the mixtures of the fields $S_{1}, S_{2}$ and $S_{3}$, two CP-odd mass eigenstates $A_{1}$ and $A_{2}$ which are composed by the fields $P_{1}$ and $P_{2}$, as well as one charged Higgs $H^{+}$. In the following, we assume $m_{h_{3}}>m_{h_{2}}>m_{h_{1}}$ and $m_{A_{2}}>m_{A_{1}}$, and call the state $h_{i}$ the SM-like Higgs boson if its dominant component comes from the field $S_{2}$.

In the NMSSM, the squared mass of the filed $S_{2}$ is given by

$$
m_{S_{2} S_{2}}^{2}=m_{Z}^{2} \cos ^{2} 2 \beta+\lambda^{2} v^{2} \sin ^{2} 2 \beta,
$$

where the last term on the right side is peculiar to any singlet extension of the MSSM [3], and its effect is to enhance the mass of the SM-like Higgs boson in comparison with the case of the MSSM. Moreover, if the inequation $m_{S_{3} S_{3}}^{2}<m_{S_{2} S_{2}}^{2}$ holds, the mixing of the field $S_{2}$ with the field $S_{3}$ in forming the SM-like Higgs boson can further enhance the mass. In this case, $h_{1}$ is a singlet-dominate scalar, $h_{2}$ acts as the SM-like Higgs boson, and due to the enhancement effects the requirement $m_{h_{2}} \simeq 125 \mathrm{GeV}$ does not necessarily need the large radiative correction of stops $[22,25]$. We remind that the singlet-dominated physical scalars (i.e. the mass eigenstates mainly composed by $S_{3}$ and $P_{2}$ respectively) are experimentally less constrained, and in case that they are lighter than about $200 \mathrm{GeV}, \mu=\lambda v_{s}$ naturally lies within the range from $100 \mathrm{GeV}$ to $200 \mathrm{GeV}$.

In practice, it is convenient to use [3]

$$
\lambda, \quad \kappa, \quad \tan \beta, \quad \mu, \quad M_{A}, \quad A_{\kappa},
$$

as input parameters, where $m_{H_{u}}^{2}, m_{H_{d}}^{2}$ and $m_{S}^{2}$ in eq. (2.2) are traded for $m_{Z}, \tan \beta$ and $\mu$ by the potential minimization conditions, and $A_{\lambda}$ is replaced by the squared mass of the CP-odd field $P_{1}$, which is given by

$$
M_{A}^{2} \equiv m_{P_{1} P_{1}}^{2}=\frac{2 \mu}{\sin 2 \beta}\left(A_{\lambda}+\kappa v_{s}\right) .
$$

Note that $M_{A}$ represents the mass scale of the new doublet $H_{1}$, and it is preferred by current experiments to be larger than about $300 \mathrm{GeV}$.

When we discuss the naturalness of the NMSSM, we consider two fine tuning quantities defined by [54]

$$
\Delta_{Z}=\max _{i}\left|\frac{\partial \log m_{Z}^{2}}{\partial \log p_{i}}\right|, \quad \Delta_{h}=\max _{i}\left|\frac{\partial \log m_{h}^{2}}{\partial \log p_{i}}\right|,
$$

where $h$ represents the SM-like Higgs boson, $p_{i}$ denotes SUSY parameters at the weak scale, and it includes the parameters listed in eq. (2.5) and top quark Yukawa coupling $Y_{t}$ with the latter used to estimate the sensitivity to stop masses. Obviously, $\Delta_{Z}\left(\Delta_{h}\right)$ measures the sensitivity of $m_{Z}\left(m_{h}\right)$ to SUSY parameters at weak scale, and the larger its value 
becomes, the more tuning is needed to get the corresponding mass. ${ }^{2}$ In our calculation, we calculate $\Delta_{Z}$ and $\Delta_{h}$ by the formulae presented in [55] and [56] respectively.

The NMSSM predicts five neutralinos, which are the mixtures of the fields Bino $\tilde{B}^{0}$, Wino $\tilde{W}^{0}$, Higgsinos $\tilde{H}_{d, u}^{0}$ and Singlino $\tilde{S}^{0}$. In the basis $\psi^{0}=\left(-i \tilde{B}^{0},-i \tilde{W}^{0}, \tilde{H}_{d}^{0}, \tilde{H}_{u}^{0}, \tilde{S}^{0}\right)$, the neutralino mass matrix is given by [3]

$$
\mathcal{M}=\left(\begin{array}{ccccc}
M_{1} & 0 & -\frac{g_{1} v_{d}}{\sqrt{2}} & \frac{g_{1} v_{u}}{\sqrt{2}} & 0 \\
& M_{2} & \frac{g_{2} v_{d}}{\sqrt{2}} & -\frac{g_{2} v_{u}}{\sqrt{2}} & 0 \\
& & 0 & -\mu & -\lambda v_{u} \\
& & & 0 & -\lambda v_{d} \\
& & & & \frac{2 \kappa}{\lambda} \mu
\end{array}\right) .
$$

If $\left|M_{1}\right|,\left|M_{2}\right| \gg|\mu|$ and $2 \kappa / \lambda \sim 1$, the Bino and Wino fields are decoupled from the rest fields. In this case, the remaining three light neutralinos $\tilde{\chi}_{i}^{0}(i=1,2,3)$ can be approximated by

$$
\tilde{\chi}_{i}^{0} \approx N_{i 3} \tilde{H}_{d}^{0}+N_{i 4} \tilde{H}_{u}^{0}+N_{i 5} \tilde{S}^{0}
$$

where the elements of the rotation matrix $N$ roughly satisfy

$$
N_{i 3}: N_{i 4}: N_{i 5} \simeq \lambda\left(v_{d} \mu-v_{u} m_{\tilde{\chi}_{i}^{0}}\right): \lambda\left(v_{u} \mu-v_{d} m_{\tilde{\chi}_{i}^{0}}\right):\left(m_{\tilde{\chi}_{i}^{0}}^{2}-\mu^{2}\right)
$$

with $m_{\tilde{\chi}_{i}^{0}}$ denoting the mass of $\tilde{\chi}_{i}^{0}$. In the following, we are interested in the parameter region featured by $\tan \beta \sim 10,2 \kappa / \lambda \sim(1-1.5)$ and $|\mu| \sim(100-200) \mathrm{GeV}$, which is hereafter dubbed as the NS scenario with $\tilde{\chi}_{1}^{0}$ being Higgsino-dominated. From eq. (2.9) and eq. (2.10), one can conclude that this scenario has following characters

- The lightest two neutralinos $\tilde{\chi}_{1}^{0}$ and $\tilde{\chi}_{2}^{0}$ are Higgsino-dominated, and $\tilde{\chi}_{3}^{0}$ is Singlinodominated. Their masses should satisfy following relations: $m_{\tilde{\chi}_{1}^{0}}<|\mu|, m_{\tilde{\chi}_{2}^{0}} \sim|\mu|$ and $m_{\tilde{\chi}_{3}^{0}}>\left|\frac{2 \kappa}{\lambda} \mu\right|>|\mu|$.

- As far as $\tilde{\chi}_{1}^{0}$ is concerned, its largest component comes from $\tilde{H}_{u}^{0}$ field. If the splitting between $m_{\tilde{\chi}_{1}^{0}}$ and $|\mu|$ is significant, its Singlino component may also be quite large. The importance of the Singlino component is that it can dilute the couplings of $\tilde{\chi}_{1}^{0}$ with $W$ and $Z$ bosons, Higgs scalars and SM fermions, and consequently the density of $\tilde{\chi}_{1}^{0}$ can coincide with the DM density measured by WMAP and Planck experiments.

- The $\tilde{H}_{u}^{0}$ and $\tilde{H}_{d}^{0}$ components in $\tilde{\chi}_{2}^{0}$ should be comparable, and they are usually much larger than $\tilde{S}^{0}$ component of $\tilde{\chi}_{2}^{0}$, i.e. $\left|N_{23}\right| \sim\left|N_{24}\right| \gg\left|N_{25}\right|$.

- As for $\tilde{\chi}_{3}^{0}$, the relation $\left|N_{35}\right|>\left|N_{33}\right|>\left|N_{34}\right|$ usually holds.

\footnotetext{
${ }^{2}$ As was pointed out in [54], if the NMSSM is considered as the low energy realization of an (unknown) overarching ultimate theory, $\Delta_{Z}$ and $\Delta_{h}$ can be thought of as providing a lower bound on electroweak fine-tuning. Any parameter point with a low $\Delta_{Z}$ and $\Delta_{h}$ implies that the ultimate theory may be low fine-tuned at high energy scale. By contrast, if the point correspond to large $\Delta_{Z}$ and $\Delta_{h}$, the underlying theory must be fine-tuned.
} 


\subsection{Strategy in scanning the parameter space of the NMSSM}

In this part, we perform a comprehensive scan over the parameter space of the NMSSM by considering various experimental constraints. Especially, we take great pains to implement the constraints from the direct searches for SUSY at the LHC. After this procedure, we get the NS scenario with a Higgsino-dominated $\tilde{\chi}_{1}^{0}$.

We begin our study by making following assumptions about some unimportant SUSY parameters:

- We fix all soft breaking parameters for the first two generation squarks at $2 \mathrm{TeV}$. Considering that the third generation squarks can affect significantly the mass of the SM-like Higgs boson, we vary freely all soft parameters in this sector except that we assume $m_{U_{3}}=m_{D_{3}}$ for right-handed soft breaking masses and $A_{t}=A_{b}$ for soft breaking trilinear coefficients.

- Considering that we require the NMSSM to explain the discrepancy of the measured value of the muon anomalous magnetic moment from its SM prediction, we treat the common value for all soft breaking parameters in the slepton sector (denoted by $m_{\tilde{l}}$ hereafter) as a free parameter.

- We fix gluino mass at $2 \mathrm{TeV}$, and treat the Bino mass $M_{1}$ and the Wino mass $M_{2}$ as free parameters since they affect the properties of the neutralinos.

Then we use the package NMSSMTools-4.9.0 $[57,58]$ to scan following parameter space:

$$
\begin{aligned}
& 0<\lambda \leq 0.75, \quad 0<\kappa \leq 0.75, \quad 2 \leq \tan \beta \leq 60, \quad 100 \mathrm{GeV} \leq m_{\tilde{l}} \leq 1 \mathrm{TeV}, \\
& 100 \mathrm{GeV} \leq \mu \leq 1 \mathrm{TeV}, \quad 50 \mathrm{GeV} \leq M_{A} \leq 2 \mathrm{TeV}, \quad\left|A_{\kappa}\right| \leq 2 \mathrm{TeV}, \\
& 100 \mathrm{GeV} \leq M_{Q_{3}}, M_{U_{3}} \leq 2 \mathrm{TeV}, \quad\left|A_{t}\right| \leq \min \left(3 \sqrt{M_{Q_{3}}^{2}+M_{U_{3}}^{2}}, 5 \mathrm{TeV}\right), \\
& 20 \mathrm{GeV} \leq M_{1} \leq 500 \mathrm{GeV}, \quad 100 \mathrm{GeV} \leq M_{2} \leq 1 \mathrm{TeV},
\end{aligned}
$$

where all the parameters are defined at the scale of $1 \mathrm{TeV}$. During the scan, we use following constraints to select physical parameter points:

(1) All the constraints implemented in the package NMSSMTools-4.9.0, which include the $Z$-boson invisible decay, the LEP search for sparticles (i.e. the lower bounds on various sparticle masses and the upper bounds on the chargino/neutralino pair production rates), the $B$-physics observables such as the branching ratios for $B \rightarrow X_{s} \gamma$ and $B_{s} \rightarrow$ $\mu^{+} \mu^{-}$, the discrepancy of the muon anomalous magnetic moment, the dark matter relic density and the LUX limits on the scattering rate of dark matter with nucleon. In getting the constraint from a certain observable which has an experimental central value, we use its latest measured result and require the NMSSM to explain the result at $2 \sigma$ level.

(2) Constraints from the direct searches for Higgs bosons at LEP, Tevatron and LHC. Especially we require that one CP-even Higgs boson acts as the SM-like Higgs boson 
discovered at the LHC. We implement these constraints with the packages HiggsSignal for $125 \mathrm{GeV}$ Higgs data fit $[59-61]^{3}$ and HiggsBounds for non-standard Higgs boson search at colliders $[64,65]$.

(3) Constraints from the fine-tuning consideration: $\Delta_{Z} \leq 50$ and $\Delta_{h} \leq 50$.

(4) Constraints from the preliminary analyses of the ATLAS and CMS groups in their direct searches for sparticles at the LHC Run-I. We implement these constraints by the packages FastLim [66] and SModelS [67]. These two packages provide cut efficiencies or upper bounds on some sparticle production processes in simplified model framework, and thus enable us to impose the direct search bounds in an easy and fast way. In the appendix, we briefly introduce the two packages.

(5) Constraints from the latest searches for electroweakinos and stops by the ATLAS collaboration at the LHC Run-I. We implement these constraints by detailed Monte Carlo simulation. Since we have to treat more than twenty thousand samples at this step, this process is rather time consuming in our calculation by clusters. In the appendix, we provide details of our simulation.

After analyzing the samples that survive the constraints, we find that they can be classified into four types: for Type I samples, $h_{1}$ corresponds to the SM-like Higgs boson and $\tilde{\chi}_{1}^{0}$ is Bino-dominated, while for Type II, III and IV samples, $h_{2}$ acts as the SM-like Higgs boson with $\tilde{\chi}_{1}^{0}$ being Bino-, Singlino- and Higgsino-dominated respectively. In table 1, we list the favored parameter ranges for each type of samples before and after considering the constraints from the direct search experiments, i.e. the constraints (4) and (5). Note that in the last row of this table, the retaining ratio $R$ is defined by $R=N_{\text {after }} / N_{\text {before }}$ where $N_{\text {before }}$ is the number of the samples that satisfy the constraints (1), (2) and (3) in our scan, and $N_{\text {after }}$ is the number of the samples that further satisfy the constraints (4) and (5). This table indicates that although the direct search experiments are effective in excluding parameter points encountered in the scan, they scarcely change the ranges of the input parameters where $\Delta_{Z}$ and $\Delta_{h}$ take rather low values, or equivalently speaking the NMSSM can naturally predict $m_{Z}$ and $m_{h}$ even after considering the direct search constraints from LHC Run-I. The underlying reason for this phenomenology is that the exclusion capability of the direct search experiments depends not only on sparticle production rate, but also on the decay chain of the sparticle and the mass gap between the sparticle and its decay product. We checked that a large portion of the excluded samples are characterized by $M_{2} \leq 250 \mathrm{GeV}$. In this case, there exist one moderately light neutralino and one moderately light chargino with both of them being Wino-dominated, and their associated production rate at the LHC is quite large so that the $3 l+E_{T}^{\text {miss }}$ signal of the production after cuts may exceed its experimental upper bound (see appendix for more information). Among the four types of points, we also find that the lowest fine-tuning comes from type III and

\footnotetext{
${ }^{3}$ In our fit, we adopt a moderately wider range of the SM-like Higgs boson mass, $122 \mathrm{GeV} \leq m_{h} \leq$ $128 \mathrm{GeV}$, in comparison with the default uncertainty of $2 \mathrm{GeV}$ for $m_{h}$ in the package HiggsSignal. This is because $\lambda \gtrsim 0.4$ may induce a $\mathcal{O}(1 \mathrm{GeV})$ correction to $m_{h}$ at two-loop level [62,63], which is not considered in the NMSSMTools.
} 


\begin{tabular}{|c|c|c|c|c|}
\hline & Type I & Type II & Type III & Type IV \\
\hline \multirow{2}{*}{$\lambda$} & $0 . \sim 0.41$ & $0 . \sim 0.68$ & $0 . \sim 0.75$ & $0.18 \sim 0.71$ \\
\hline & $0 . \sim 0.41$ & $0 . \sim 0.68$ & $0 . \sim 0.75$ & $0.18 \sim 0.71$ \\
\hline \multirow{2}{*}{$\kappa$} & $0 . \sim 0.66$ & $0 . \sim 0.52$ & $0 . \sim 0.27$ & $0 . \sim 0.51$ \\
\hline & $0 . \sim 0.66$ & $0 . \sim 0.52$ & $0 . \sim 0.27$ & $0 . \sim 0.51$ \\
\hline \multirow{2}{*}{$\tan \beta$} & $3 \sim 60$ & $4 \sim 38$ & $3 \sim 60$ & $3 \sim 18$ \\
\hline & $3 \sim 60$ & $4 \sim 38$ & $3 \sim 60$ & $3 \sim 18$ \\
\hline \multirow{2}{*}{$\mu(\mathrm{GeV})$} & $150 \sim 400$ & $115 \sim 370$ & $105 \sim 315$ & $110 \sim 175$ \\
\hline & $180 \sim 400$ & $115 \sim 370$ & $105 \sim 315$ & $110 \sim 165$ \\
\hline \multirow{2}{*}{$A_{\kappa}(\mathrm{GeV})$} & $-2000 \sim 0$ & $-750 \sim 0$ & $-350 \sim 40$ & $-650 \sim-20$ \\
\hline & $-2000 \sim 0$ & $-750 \sim 0$ & $-350 \sim 40$ & $-650 \sim-30$ \\
\hline \multirow{2}{*}{$M_{Q_{3}}(\mathrm{GeV})$} & $390 \sim 2000$ & $550 \sim 2000$ & $450 \sim 2000$ & $450 \sim 2000$ \\
\hline & $570 \sim 2000$ & $680 \sim 2000$ & $450 \sim 2000$ & $620 \sim 2000$ \\
\hline \multirow{2}{*}{$M_{U_{3}}(\mathrm{GeV})$} & $480 \sim 2000$ & $530 \sim 2000$ & $480 \sim 2000$ & $490 \sim 2000$ \\
\hline & $610 \sim 2000$ & $680 \sim 2000$ & $580 \sim 2000$ & $560 \sim 2000$ \\
\hline \multirow{2}{*}{$M_{\tilde{l}}(\mathrm{GeV})$} & $100 \sim 1000$ & $100 \sim 640$ & $100 \sim 730$ & $100 \sim 950$ \\
\hline & $100 \sim 1000$ & $100 \sim 640$ & $100 \sim 730$ & $100 \sim 950$ \\
\hline \multirow{2}{*}{$A_{t}(\mathrm{GeV})$} & $-4700 \sim 5000$ & $-4500 \sim 4500$ & $-5000 \sim 4800$ & $-5000 \sim 4500$ \\
\hline & $-4400 \sim 4850$ & $-4500 \sim 4500$ & $-5000 \sim 4600$ & $-5000 \sim 4500$ \\
\hline \multirow{2}{*}{$M_{1}(\mathrm{GeV})$} & $40 \sim 350$ & $20 \sim 175$ & $45 \sim 500$ & $120 \sim 500$ \\
\hline & $40 \sim 350$ & $40 \sim 175$ & $45 \sim 500$ & $120 \sim 500$ \\
\hline \multirow{2}{*}{$M_{2}(\mathrm{GeV})$} & $105 \sim 1000$ & $150 \sim 1000$ & $150 \sim 1000$ & $160 \sim 1000$ \\
\hline & $105 \sim 1000$ & $150 \sim 1000$ & $155 \sim 1000$ & $165 \sim 1000$ \\
\hline \multirow{2}{*}{$M_{A}(\mathrm{GeV})$} & $200 \sim 2000$ & $900 \sim 2000$ & $500 \sim 2000$ & $430 \sim 2000$ \\
\hline & $320 \sim 2000$ & $900 \sim 2000$ & $500 \sim 2000$ & $430 \sim 2000$ \\
\hline$R$ & $52 \%$ & $54 \%$ & $71 \%$ & $65 \%$ \\
\hline
\end{tabular}

Table 1. Favored parameter ranges for different types of samples. In each item, the range in the first row is for the samples that survive the constraints (1), (2) and (3) presented in the text, and that in the second row corresponds to the samples that further satisfy the constraints from the direct search for sparticles at the LHC Run-I, i.e. the constraints (4) and (5). The quantity $R$ in the last row represents the retaining ratio of the samples before and after considering the direct search constraints in our scan. For Type I samples, $h_{1}$ corresponds to the SM-like Higgs boson and $\tilde{\chi}_{1}^{0}$ is Bino-dominated, while for Type II, III and IV samples, $h_{2}$ acts as the SM-like Higgs boson with $\tilde{\chi}_{1}^{0}$ being Bino-, Singlino- and Higgsino-dominated respectively.

type IV samples, for which $\Delta_{Z}$ and $\Delta_{h}$ may be as low as about 2 . This character is shown in figure 1, where we project type III and IV samples on $\Delta_{Z}-\Delta_{h}$ plane. We emphasize that for the type IV samples with $\Delta_{Z}, \Delta_{h} \lesssim 10, \mu$ is upper bounded by about $145 \mathrm{GeV}$. In this case, it is compressed spectrum among the Higgsino-dominated particles $\tilde{\chi}_{1}^{0}, \tilde{\chi}_{2}^{0}$ and $\tilde{\chi}_{1}^{ \pm}$that helps the samples evade the direct search experiments.

Because the type IV samples were scarcely studied in previous literatures and also because they have similar phenomenology to that of the NS scenario in the MSSM, we in the following focus on this type of samples. In order to make the essential features of the 

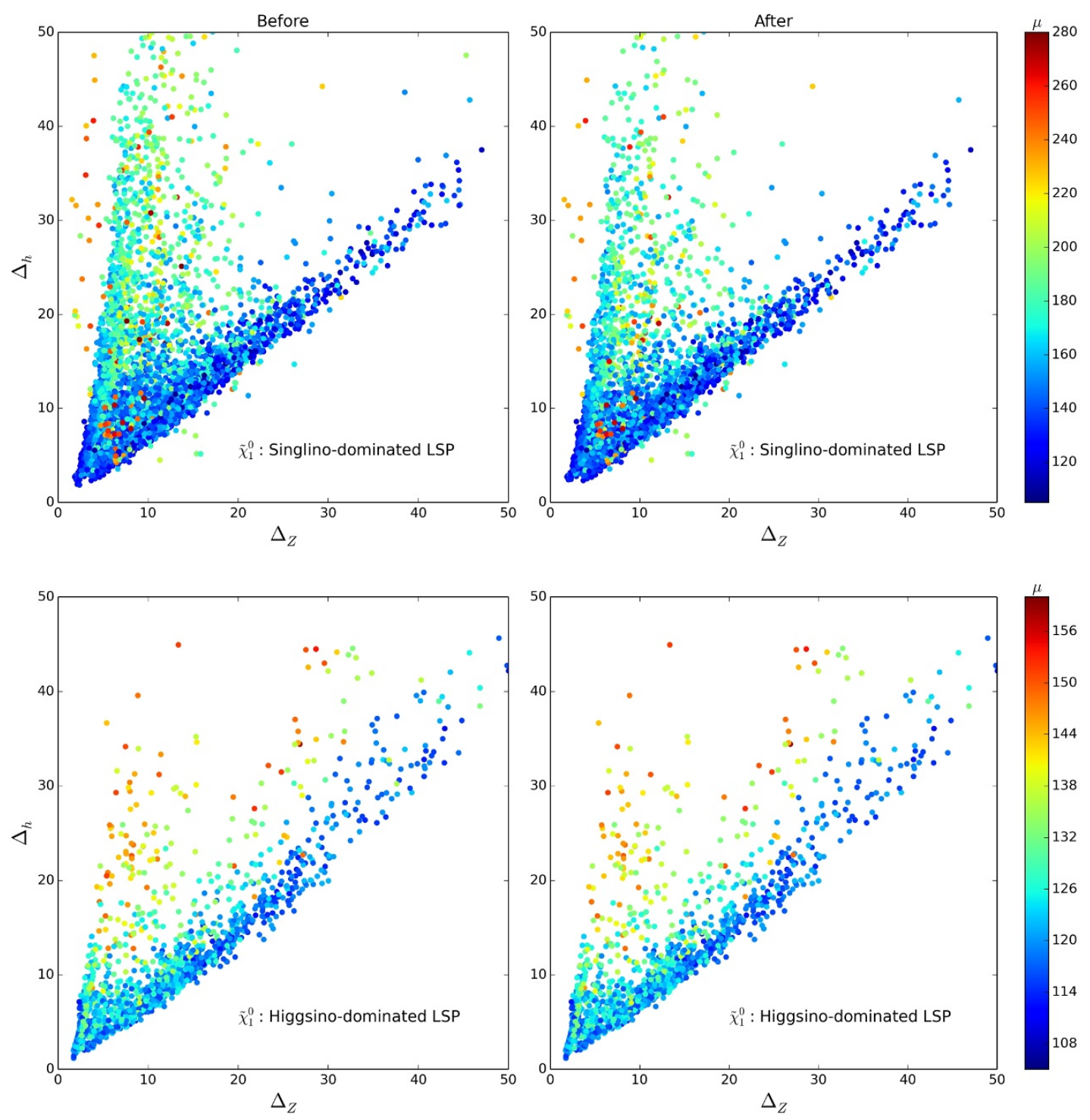

Figure 1. $\Delta_{Z}$ and $\Delta_{h}$ predicted by the Type III samples (upper panels) and Type IV samples (lower panels) respectively. Samples in the left panels survive the constraints (1), (2) and (3) presented in the text, and those in the right panels further satisfy the constraints from the direct searches for sparticles at LHC Run I, i.e. the constraints (4) and (5). The panels in each row adopt same color convention for $\mu$, which is presented on the right side of the row.

samples clear, we only consider those that satisfy additionally the condition $M_{1}, M_{2}, m_{\tilde{l}} \geq$ $300 \mathrm{GeV}$. Hereafter we call such samples collectively as the NS scenario with a Higgsinodominated $\tilde{\chi}_{1}^{0}$. As we will show below, $|\mu|$ in this scenario is upper bounded by about $160 \mathrm{GeV}$, so the condition is equivalent to $M_{1}, M_{2}, m_{\tilde{l}} \gtrsim 2|\mu|$. In this case, gauginos and sleptons affect little on the properties of the lightest three neutralino, and the lighter chargino. 


\begin{tabular}{|c|c|c|c|c|c|}
\hline Para & Range & ara & Range & Para & Range \\
\hline \multirow{2}{*}{$\tan \beta$} & $7 \sim 18$ & \multirow[b]{2}{*}{$m_{\tilde{\chi}_{1}^{0}}$} & 585 & \multirow{2}{*}{$A_{\kappa}$} & $-400 \sim-$ \\
\hline & $7 \sim$ & & $65 \sim 85$ & & $-400 \sim$ \\
\hline \multirow{2}{*}{1} & $0.15 \sim 0$. & \multirow{2}{*}{$m_{\tilde{\chi}_{2}^{0}}$} & $125 \sim 195$ & \multirow[b]{2}{*}{$m_{h_{1}}$} & $45 \sim$ \\
\hline & $0.15 \sim$ & & $125 \sim$ & & $45 \sim$ \\
\hline & $0.28 \sim$ & \multirow{2}{*}{$m_{\tilde{\chi}_{3}^{0}}$} & $150 \sim 260$ & \multirow{2}{*}{$m_{A_{1}}$} & $120 \sim$ \\
\hline & $0.28 \sim 0.68$ & & $150 \sim 260$ & & $120 \sim$ \\
\hline \multirow[b]{2}{*}{$\mu$} & $110 \sim 160$ & \multirow{2}{*}{$m_{\tilde{\chi}_{1}^{ \pm}}$} & $105 \sim 150$ & \multirow{2}{*}{$m_{H^{ \pm}}$} & $800 \sim 2000$ \\
\hline & $110 \sim 160$ & & $105 \sim 150$ & & $800 \sim 2000$ \\
\hline & $-5000 \sim 4500$ & \multirow{2}{*}{$m_{\tilde{t}_{1}}$} & $380 \sim 2050$ & \multirow{2}{*}{$m_{\tilde{t}_{2}}$} & $1050 \sim 3100$ \\
\hline & $-5000 \sim 4500$ & & $500 \sim 2050$ & & $1100 \sim 3100$ \\
\hline
\end{tabular}

Table 2. Similar to table 1, but for the NS scenario of the NMSSM with $\tilde{\chi}_{1}^{0}$ being Higgsino dominant. This scenario is defined by Type IV samples, and it further requires the samples to satisfy $M_{1}, M_{2}, m_{\tilde{l}} \geq 300 \mathrm{GeV}$. Quantities with mass dimension are in unit of $\mathrm{GeV}$.

\section{Key features of the NS scenario with $\tilde{\chi}_{1}^{0}$ being Higgsino-dominated}

In this section, we investigate the features of the NS scenario with $\tilde{\chi}_{1}^{0}$ being Higgsinodominated. We are particulary interested in neutralino-chargino sector and stop sector since they play an important role in determining the fine tunings of the theory. In table 2 , we show the favored ranges of some quantities such as $\mu$ and stop masses. This table indicates that our scenario is featured by $\mu / \mathrm{GeV} \in[110,160], m_{\tilde{\chi}_{1}^{0}} / \mathrm{GeV} \in[65,85], m_{\tilde{\chi}_{2}^{0}} / \mathrm{GeV} \in$ $[125,195], m_{\tilde{\chi}_{3}^{0}} / \mathrm{GeV} \in[150,260], m_{\tilde{\chi}_{1}^{ \pm}} / \mathrm{GeV} \in[105,150]$ and $m_{\tilde{t}_{1}} / \mathrm{GeV} \in[500,2050]$, and among the ranges, only the lower bound of $m_{\tilde{t}_{1}}$ is shifted from $380 \mathrm{GeV}$ to $500 \mathrm{GeV}$ by the constraints from the direct search experiments. Moreover, we checked that in our scenario, the ratio $2 \kappa / \lambda$ is restricted in the range from about 1 to 1.5. In this case, Higgsinos and Singlino are approximately degenerated in mass, and consequently they mix strongly to form mass eigenstates.

In figure 2 , we show the mass spectrum of $\tilde{\chi}_{1}^{0}, \tilde{\chi}_{2}^{0}, \tilde{\chi}_{3}^{0}$ and $\tilde{\chi}_{1}^{ \pm}$in our scenario. This figure indicates that the mass splittings among the particles satisfy $30 \mathrm{GeV} \lesssim \Delta_{ \pm} \lesssim 70 \mathrm{GeV}$, $50 \mathrm{GeV} \lesssim \Delta_{0} \lesssim 110 \mathrm{GeV}$ and $80 \mathrm{GeV} \lesssim\left(m_{\tilde{\chi}_{3}^{0}}-m_{\tilde{\chi}_{1}^{0}}\right) \lesssim 160 \mathrm{GeV}$. We remind that these splittings are induced by the strong mixings between Higgsinos and Singlino, and significantly larger than those among $\tilde{\chi}_{1}^{0}, \tilde{\chi}_{2}^{0}$ and $\tilde{\chi}_{1}^{ \pm}$in the NS scenario of the MSSM [5]. In figure 3 , we show the field components of the states $\tilde{\chi}_{1}^{0}, \tilde{\chi}_{2}^{0}$ and $\tilde{\chi}_{3}^{0}$ respectively. As is expected, the $\tilde{H}_{u}^{0}, \tilde{S}^{0}$ and $\tilde{H}_{d}^{0}$ components in $\tilde{\chi}_{1}^{0}$ are comparable in magnitude with the largest one coming from the $\tilde{H}_{u}^{0}$ component. We emphasize again that the large Singlino component, i.e. $N_{15} \sim 0.5$, can dilute the interactions of the Higgsino-dominated $\tilde{\chi}_{1}^{0}$ with other fields, and consequently $\tilde{\chi}_{1}^{0}$ can reach its right relic density. In this case, we checked that the main annihilation channels of $\tilde{\chi}_{1}^{0}$ in early universe include $\tilde{\chi}_{1}^{0} \tilde{\chi}_{1}^{0} \rightarrow$ $W^{+} W^{-}, Z Z, Z h_{1}, h_{1} h_{1}, h_{1} h_{2}, q \bar{q}$. As for $\tilde{\chi}_{2}^{0}$, its largest component comes from either $\tilde{H}_{d}^{0}$ field (for most cases) or $\tilde{H}_{u}^{0}$ field (in rare cases), and in general the two components are comparable, which can be learned from the figure and also from eq. (2.10). We checked 

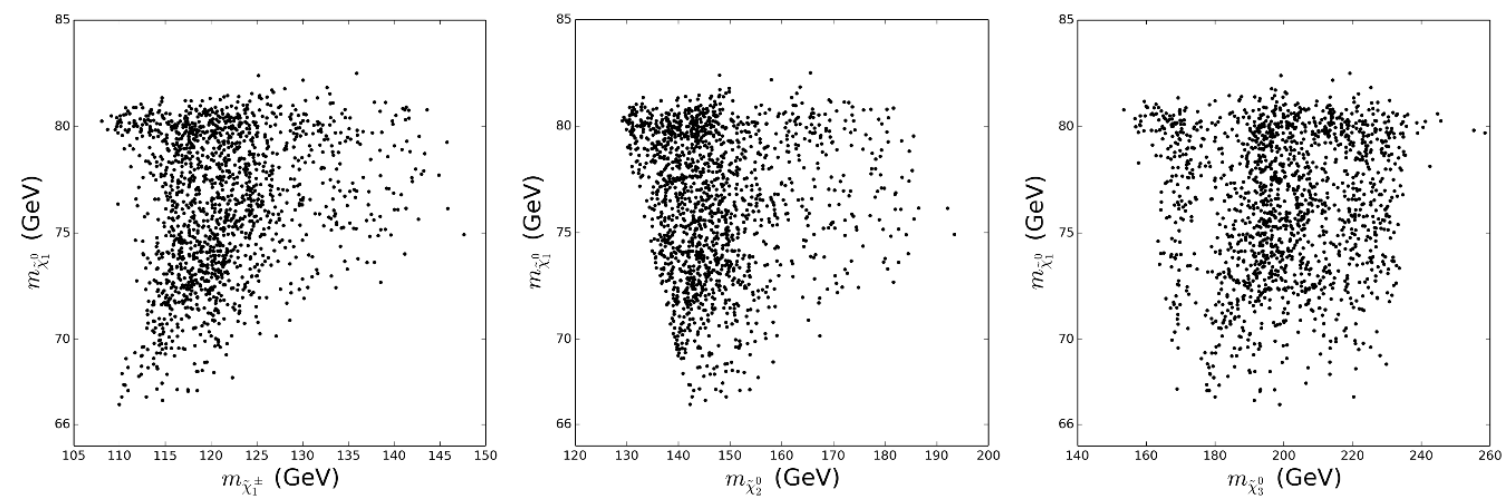

Figure 2. Mass spectrum of $\tilde{\chi}_{1}^{0}, \tilde{\chi}_{2}^{0}, \tilde{\chi}_{3}^{0}$ and $\tilde{\chi}_{1}^{ \pm}$in the NS scenario with $\tilde{\chi}_{1}^{0}$ being Higgsinodominated. Note that the mass splittings among these particles are induced by the strong mixings between Higgsinos and Singlino, and significantly larger than those in the NS scenario of the MSSM.
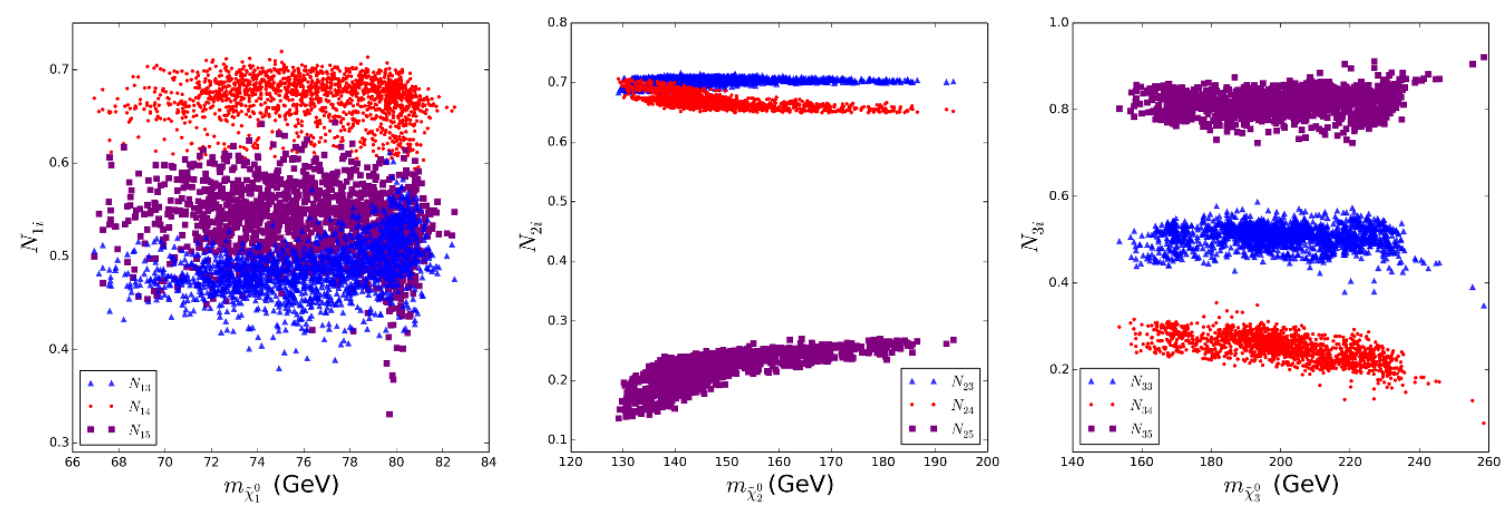

Figure 3. Components of the physical states $\tilde{\chi}_{1}^{0}, \tilde{\chi}_{2}^{0}$ and $\tilde{\chi}_{3}^{0}$ in the NS scenario with $\tilde{\chi}_{1}^{0}$ being Higgsino-dominated. Note that these results can be understood by eq. (2.10).

that due to the spectrum and the mixings, the dominant decay of $\tilde{\chi}_{1}^{ \pm}$is $\tilde{\chi}_{1}^{ \pm} \rightarrow \tilde{\chi}_{1}^{0} W^{*}$, and that of $\tilde{\chi}_{2}^{0}$ is usually $\tilde{\chi}_{2}^{0} \rightarrow \tilde{\chi}_{1}^{0} Z^{(*)}$. By contrast, the possible dominant decay modes of $\tilde{\chi}_{3}^{0}$ are rather rich, which include $\tilde{\chi}_{1}^{0} Z^{(*)}, \tilde{\chi}_{1}^{0} h_{1}, \tilde{\chi}_{1}^{ \pm} W^{(*)}$. Since $\tilde{\chi}_{3}^{0}$ is Singlino-dominated, its production rate is rather low, and consequently its phenomenology is of less interest.

Next we turn to the properties of $\tilde{t}_{1}$. From the interactions of $\tilde{t}_{1}$ presented in [1], one can infer that if $\tilde{t}_{1}$ is $\tilde{t}_{R}$ dominated and meanwhile $\left|N_{14}\right| \simeq\left|N_{24}\right|$, the relation $\operatorname{Br}\left(\tilde{t}_{1} \rightarrow\right.$ $\left.\tilde{\chi}_{1}^{+} b\right): \operatorname{Br}\left(\tilde{t}_{1} \rightarrow \tilde{\chi}_{1}^{0} t\right): \operatorname{Br}\left(\tilde{t}_{1} \rightarrow \tilde{\chi}_{2}^{0} t\right) \simeq 2: 1: 1$ should hold. On the other hand, if $\tilde{t}_{1}$ is $\tilde{t}_{L}$ dominated, $\tilde{t}_{1}$ prefers to decay into the Higgsino-dominated $\tilde{\chi}_{1,2}^{0}$ with $\operatorname{Br}\left(\tilde{t}_{1} \rightarrow \tilde{\chi}_{1}^{0} t\right) \simeq$ $\operatorname{Br}\left(\tilde{t}_{1} \rightarrow \tilde{\chi}_{2}^{0} t\right)$. These features are exhibited in figure 4, where we show the correlations between different decay rates of $\tilde{t}_{1}$ in our scenario. From figure 4, one can also learn that the branching ratio of $\tilde{t}_{1} \rightarrow \tilde{\chi}_{3}^{0} t$ is less than $10 \%$. This is because $\tilde{\chi}_{3}^{0}$ is Singlino-dominated and its $\tilde{H}_{u}^{0}$ component is small. Moreover, we note that in our scenario $m_{\tilde{t}_{1}}$ is lower bounded by about $500 \mathrm{GeV}$, which is about $100 \mathrm{GeV}$ less than that in the NS scenario of the MSSM [68-70]. One reason for the difference is that $\tilde{t}_{1}$ in our scenario has richer decay modes. 

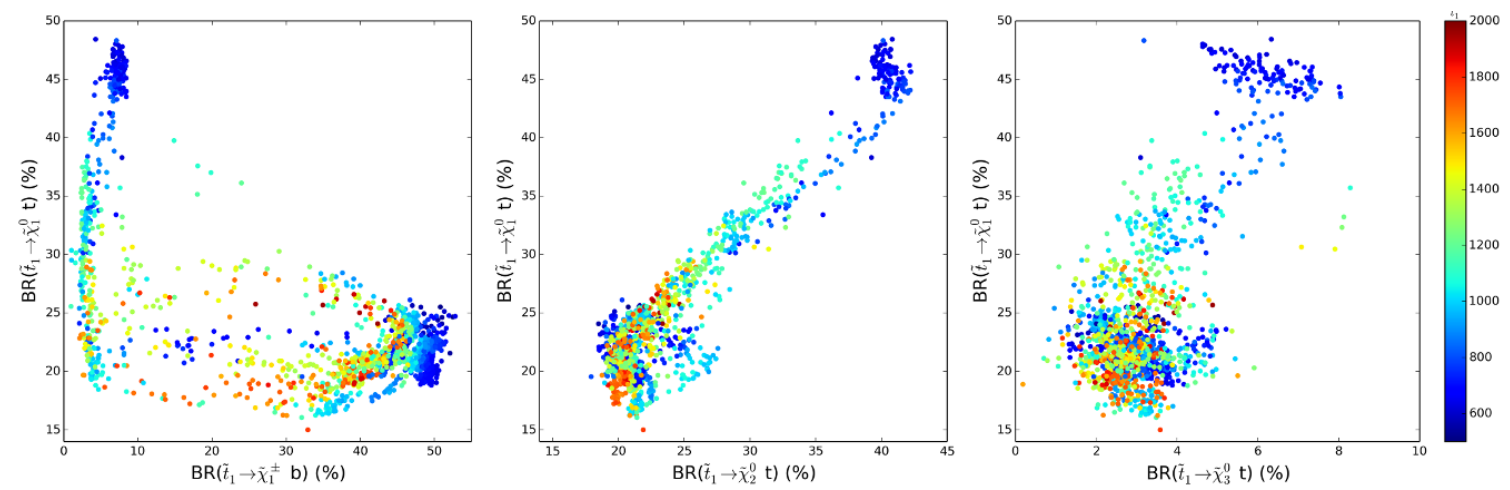

Figure 4. Correlations among the main decay modes of $\tilde{t}_{1}$ in the NS scenario with $\tilde{\chi}_{1}^{0}$ being Higgsino-dominated. Note that if $\tilde{t}_{1}$ is $\tilde{t}_{R}$ dominated and meanwhile $\left|N_{14}\right| \simeq\left|N_{24}\right|$, we have $\operatorname{Br}\left(\tilde{t}_{1} \rightarrow\right.$ $\left.\tilde{\chi}_{1}^{+} b\right): \operatorname{Br}\left(\tilde{t}_{1} \rightarrow \tilde{\chi}_{1}^{0} t\right): \operatorname{Br}\left(\tilde{t}_{1} \rightarrow \tilde{\chi}_{2}^{0} t\right) \simeq 2: 1: 1$. On the other hand, if $\tilde{t}_{1}$ is $\tilde{t}_{L}$ dominated, $\tilde{t}_{1}$ prefers to decay into the Higgsino-dominated $\tilde{\chi}_{1,2}^{0}$ with $\operatorname{Br}\left(\tilde{t}_{1} \rightarrow \tilde{\chi}_{1}^{0} t\right) \simeq \operatorname{Br}\left(\tilde{t}_{1} \rightarrow \tilde{\chi}_{2}^{0} t\right)$.

\begin{tabular}{|c|c|c|c|c|c|c|c|}
\hline & $m_{\tilde{\chi}_{1}^{0}}$ & $m_{\tilde{\chi}_{2}^{0}}$ & $m_{\tilde{\chi}_{3}^{0}}$ & $m_{\tilde{\chi}_{1}^{ \pm}}$ & $\operatorname{BR}\left(\tilde{\chi}_{2}^{0} \rightarrow \tilde{\chi}_{1}^{0} Z^{(*)}\right)$ & $\operatorname{BR}\left(\tilde{\chi}_{3}^{0} \rightarrow \tilde{\chi}_{1}^{0} Z^{(*)}\right)$ & $\operatorname{BR}\left(\tilde{\chi}_{1}^{ \pm} \rightarrow \tilde{\chi}_{1}^{0} W^{*}\right)$ \\
\hline P1 & 80.2 & 129.1 & 158.4 & 108.0 & $94.2 \%$ & $9.08 \%$ & $100 \%$ \\
\hline P2 & 67.3 & 142.6 & 180.0 & 110.2 & $94.7 \%$ & $7.55 \%$ & $100 \%$ \\
\hline P3 & 82.5 & 165.5 & 219.2 & 135.9 & $98.1 \%$ & $26.3 \%$ & $100 \%$ \\
\hline P4 & 74.9 & 193.4 & 220.6 & 147.6 & $96.2 \%$ & $6.50 \%$ & $100 \%$ \\
\hline
\end{tabular}

Table 3. Mass and decay information of the benchmark points P1, P2, P3 and P4 in our study.

\section{Future detection of our scenario}

\subsection{Detection at $14 \mathrm{TeV}$ LHC}

From the analysis in last section, one can learn that the NS scenario with $\tilde{\chi}_{1}^{0}$ being Higgsino-dominated is characterized by predicting $\mu \leq 160 \mathrm{GeV}$ and sizable mass splittings among the Higgsino-dominated neutralinos and chargino, i.e. $30 \mathrm{GeV} \leq \Delta_{ \pm} \leq 70 \mathrm{GeV}$ and $50 \mathrm{GeV} \leq \Delta_{0} \leq 110 \mathrm{GeV}$. Although this kind of spectrum is allowed by the direct searches for the electroweakinos at LHC Run I, it is expected to be tightly constrained at the upgraded LHC.

We investigate this issue by considering the neutralino and chargino associated production processes at $14 \mathrm{TeV}$ LHC. For simplicity we adopt 4 benchmark points listed in table 3 , which are discriminated by the values of $\mu$ (or equivalently $m_{\tilde{\chi}_{1}^{ \pm}}$), $\Delta_{0}$ and $\Delta_{ \pm}$. Since $\tilde{\chi}_{1}^{ \pm}$for these points decays into $\tilde{\chi}_{1}^{0}$ plus an off-shell $W$ boson, and $\tilde{\chi}_{2}^{0}$ decays mainly into $\tilde{\chi}_{1}^{0}$ plus a $Z$ boson (on-shell or off-shell), the signal region SR0 $\tau$ a in the ATLAS direct searches for electroweakinos by trileptons and large $E_{T}^{\text {miss }}$ signal [71], which was proposed in the analysis [72] and also briefly introduced in the appendix of this work, is most pertinent to explore those points. In our analysis, we simulate the processes $p p \rightarrow \tilde{\chi}_{1}^{ \pm} \tilde{\chi}_{2}^{0}, \tilde{\chi}_{1}^{ \pm} \tilde{\chi}_{3}^{0} \rightarrow 3 l+E_{T}^{\text {miss }}$ to get their summed rate in each bin of the signal region, and present the result in the last four columns of table 4 . We also present in the table the backgrounds of the bins at $14 \mathrm{TeV}$ LHC, which were obtained by detailed simulations done in [9]. With these results, 


\begin{tabular}{|c|c|c|c|c|c|c|c|c|c|}
\hline SR0 $\tau$ a & $m_{\text {SFOS }}$ & $m_{T}$ & $E_{T}^{\text {miss }}$ & $m_{3 l}$ & Background & P1 & P2 & P3 & P4 \\
\hline 1 & $12-40$ & $0-80$ & $50-90$ & no & 2.41 & 0.652 & 0.423 & 0.183 & 0.007 \\
\hline 2 & $12-40$ & $0-80$ & $>90$ & no & 0.45 & 0.273 & 0.176 & 0.108 & 0.003 \\
\hline 3 & $12-40$ & $>80$ & $50-75$ & no & 1 & 0.070 & 0.054 & 0.040 & 0.001 \\
\hline 4 & $12-40$ & $>80$ & $>75$ & no & 1.08 & 0.064 & 0.074 & 0.074 & 0.008 \\
\hline 5 & $40-60$ & $0-80$ & $50-75$ & yes & 1.37 & 0.131 & 0.365 & 0.170 & 0.006 \\
\hline 6 & $40-60$ & $0-80$ & $>75$ & no & 0.76 & 0.119 & 0.509 & 0.302 & 0.013 \\
\hline 7 & $40-60$ & $>80$ & $50-135$ & no & 1.49 & 0.122 & 0.240 & 0.183 & 0.011 \\
\hline 8 & $40-60$ & $>80$ & $>135$ & no & 0.2 & 0.008 & 0.022 & 0.022 & 0.002 \\
\hline 9 & $60-81.2$ & $0-80$ & $50-75$ & yes & 2.4 & 0.032 & 0.156 & 0.218 & 0.040 \\
\hline 10 & $60-81.2$ & $>80$ & $50-75$ & no & 1.51 & 0.027 & 0.074 & 0.087 & 0.015 \\
\hline 11 & $60-81.2$ & $0-110$ & $>75$ & no & 2.98 & 0.062 & 0.312 & 0.438 & 0.094 \\
\hline 12 & $60-81.2$ & $>110$ & $>75$ & no & 0.63 & 0.039 & 0.072 & 0.082 & 0.017 \\
\hline 13 & $81.2-101.2$ & $0-110$ & $50-90$ & yes & 66.41 & 0.024 & 0.415 & 0.146 & 0.870 \\
\hline 14 & $81.2-101.2$ & $0-110$ & $>90$ & no & 21.62 & 0.016 & 0.303 & 0.107 & 0.744 \\
\hline 15 & $81.2-101.2$ & $>110$ & $50-135$ & no & 5.98 & 0.031 & 0.086 & 0.047 & 0.157 \\
\hline 16 & $81.2-101.2$ & $>110$ & $>135$ & no & 0.59 & 0.006 & 0.018 & 0.025 & 0.032 \\
\hline 17 & $>101.2$ & $0-180$ & $50-210$ & no & 7.65 & 0.066 & 0.136 & 0.091 & 0.032 \\
\hline 18 & $>101.2$ & $>180$ & $50-210$ & no & 0.44 & 0.008 & 0.026 & 0.017 & 0.001 \\
\hline 19 & $>101.2$ & $0-120$ & $>210$ & no & 0.24 & 0.002 & 0.003 & 0.002 & 0.005 \\
\hline 20 & $>101.2$ & $>120$ & $>210$ & no & 0.09 & 0.002 & 0.005 & 0.007 & 0.000 \\
\hline
\end{tabular}

Table 4. Cross sections of the four benchmark points in each bin of the signal region SR0 $\tau$ a, which are obtained from our simulations and presented in the last four columns of this table. Quantities from the second column to the fifth column define the bins of the SR0 $\tau$ a, and their physical meanings are explained in appendix B.1. The sixth column corresponds to the backgrounds of the bins, which are taken from [9]. All quantities with mass dimension and cross sections are in units of $\mathrm{GeV}$ and $\mathrm{fb}$ respectively.

\begin{tabular}{|l|l|l|l|l|}
\hline & \multicolumn{2}{|c|}{$30 \mathrm{fb}^{-1}$} & \multicolumn{2}{c|}{$300 \mathrm{fb}^{-1}$} \\
\hline P1 & $S(\operatorname{bin} 2)=2.09$ & $S(\operatorname{bin} 1)=1.75$ & $S(\operatorname{bin} 2)=4.59$ & $S(\operatorname{bin} 1)=2.54$ \\
\hline P2 & $S(\operatorname{bin} 6)=2.88$ & $S(\operatorname{bin} 5)=1.44$ & $S(\operatorname{bin} 6)=5.58$ & $S(\operatorname{bin} 2)=2.96$ \\
\hline P3 & $S(\operatorname{bin} 6)=1.71$ & $S(\operatorname{bin} 11)=1.01$ & $S(\operatorname{bin} 6)=3.31$ & $S(\operatorname{bin} 2)=1.82$ \\
\hline P4 & $S(\operatorname{bin} 14)=0.32$ & $S(\operatorname{bin} 15)=0.21$ & $S(\operatorname{bin} 16)=0.42$ & $S(\operatorname{bin} 14)=0.34$ \\
\hline
\end{tabular}

Table 5. The best two signal bins and corresponding significances for the benchmark points with $30 \mathrm{fb}^{-1}$ and $300 \mathrm{fb}^{-1}$ integrated luminosity data respectively at $14 \mathrm{TeV}$ LHC.

we evaluate the significance $S=s / \sqrt{b+(\epsilon b)^{2}}$ for each bin, where $s$ and $b$ correspond to the number of signal and background events and $\epsilon=10 \%$ is the assumed systematical uncertainty of the backgrounds. Assuming $30 \mathrm{fb}^{-1}$ and $300 \mathrm{fb}^{-1}$ integrated luminosity data at $14 \mathrm{TeV}$ LHC, we present the best two signal bins and corresponding expected significances for P1, P2, P3 and P4 in table 5. This table reveals following information: 
- With $30 \mathrm{fb}^{-1}$ integrated luminosity data, $\mathrm{P} 1$ and $\mathrm{P} 2$ can be excluded at $2 \sigma$ confidence level, and with $300 \mathrm{fb}^{-1}$ data P3 can also be excluded. In any case, the point P4 is hard to be excluded.

- For each point, which signal bin is best for exclusion depends on the mass splittings among the neutralinos and chargino. For example, since $\Delta_{ \pm}<m_{W}$ for all the four points, the most effective bins usually require $m_{T}<80$ or $110 \mathrm{GeV}$. For points P1, P2 and P3, the bins satisfying $m_{\mathrm{SFOS}}<m_{Z}$ are preferred for exclusion since $\Delta_{0}<m_{Z}$, and by contrast the bins with $\left|m_{\mathrm{SFOS}}-m_{Z}\right|<10 \mathrm{GeV}$ (such as bins 14 and 15) are favored by point $\mathrm{P} 4$ since in this case $\tilde{\chi}_{2}^{0}$ can decay into an on-shell $Z$ boson. Note that for bins 14 and 15, the backgrounds are relatively large, and that is why the point $\mathrm{P} 4$ can not be excluded at $14 \mathrm{TeV}$ LHC after including the systematic uncertainties.

- With $300 \mathrm{fb}^{-1}$ data, the point P2 can be discovered at $14 \mathrm{TeV}$ LHC. This is partially because $\tilde{\chi}_{2}^{0}$ and $\tilde{\chi}_{1}^{ \pm}$are relatively light so that the rate of their associated production is large, and also because they have sizable mass splittings from $\tilde{\chi}_{1}^{0}$ to result in moderately energetic decay products.

- Since the bins in the SR0 $\tau$ a are disjoint, in principle their results can be statistically combined to maximize the significance. We did this, but we found that the improvement is not significant.

Since the four benchmark points stand for the typical situation in our scenario, we conclude that the future LHC experiments can exclude most part of the parameter space for the scenario, and consequently the fine-tuning of the NMSSM can be pushed to higher level. We will discuss such an issue extensively in our forthcoming work.

\subsection{Dark matter direct search}

Since $\tilde{\chi}_{1}^{0}$ as the DM candidate has quite large $\tilde{H}_{u}^{0}, \tilde{H}_{d}^{0}$ and $\tilde{S}^{0}$ components in our scenario, its interactions with the Higgs bosons and $Z$ boson are unsuppressed. As a result, the spin-independent (SI) and spin-dependent (SD) cross sections of the $\tilde{\chi}_{1}^{0}$-nucleon scattering are sizable, and may reach the sensitivities of future DM direct detection experiments such as XENON-1T and LZ-7.2T experiments [73]. In this section, we investigate such an issue. In figure 5 , we project the samples of our scenario on $m_{\tilde{\chi}_{1}^{0}}-\sigma_{\tilde{\chi}-p}^{\mathrm{SI}}$ and $m_{\tilde{\chi}_{1}^{0}}-\sigma_{\tilde{\chi}-p}^{\mathrm{SD}}$ planes with $\sigma_{\tilde{\chi}-p}^{\mathrm{SI}}$ and $\sigma_{\tilde{\chi}-p}^{\mathrm{SD}}$ denoting the SI and SD cross sections respectively. The blue lines, red lines and green lines are the sensitivities of LUX, XENON-1T and LZ experiments respectively.

For the SI cross section, one can learn from figure 5 that the future XENON-1T experiment is able to probe a large portion of the samples, and the LZ experiment can test even more. Anyhow, there still exist some samples remaining untouched by these future experiments. We numerically checked that for the untouched samples, there exists rather strong cancelation among the contributions induced by different CP-even Higgs bosons. On the other hand, the story for $\sigma_{\tilde{\chi}-p}^{\mathrm{SD}}$ is quite different. From the right panel of figure 5 one can see that the future XENON-1T experiment can test nearly all of the samples, let 

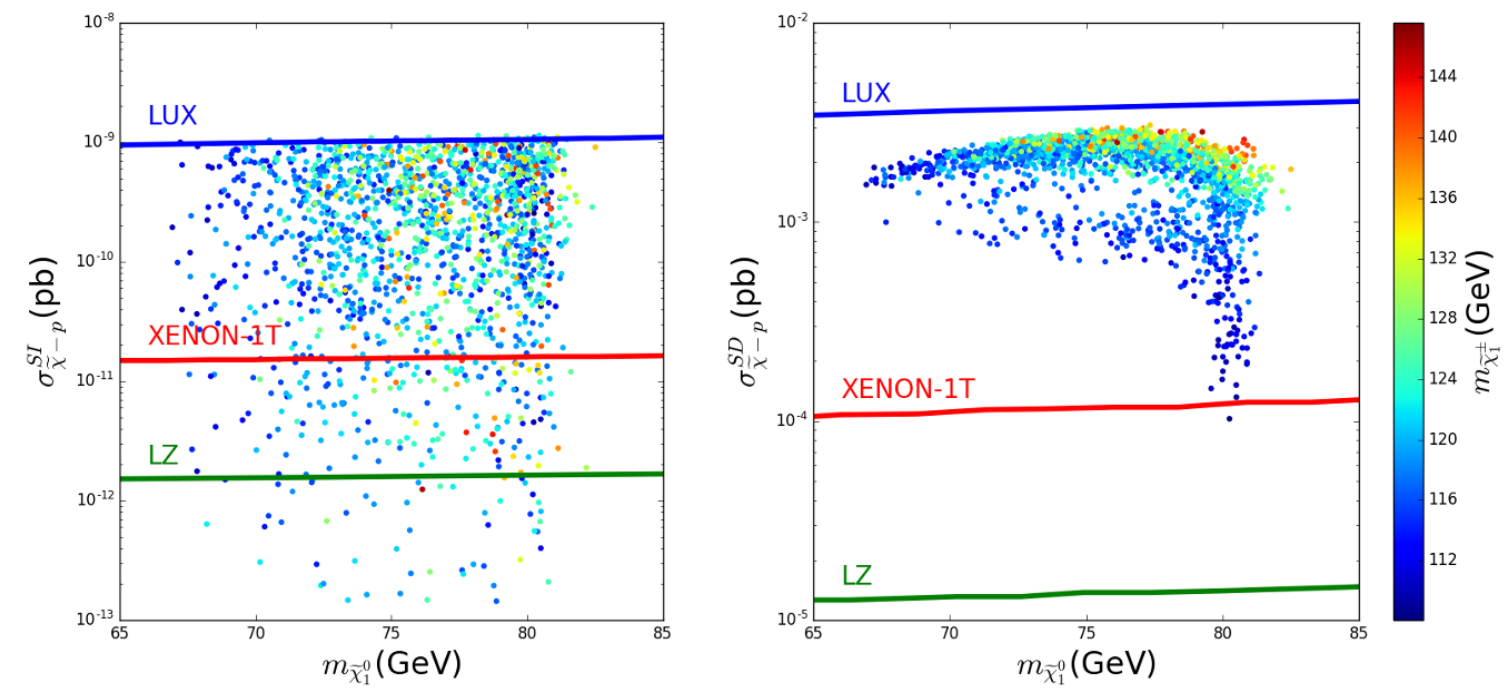

Figure 5. Spin-independent (SI) and Spin-dependent (SD) DM-nucleon scattering cross sections versus DM mass in the NS scenario with $\tilde{\chi}_{1}^{0}$ being Higgsino-dominated. Capabilities of future DM direct detection experiments in detecting the scattering are also plotted.

alone the more sensitive LZ experiment. The underlying reason is that in the NMSSM with heavy sfermions, the SD cross section gets contribution mainly from the $t$-channel $Z$-mediated diagram. As a result, the size of the cross section is determined by the $Z \tilde{\chi}_{1}^{0} \tilde{\chi}_{1}^{0}$ coupling, which is given by

$$
g_{Z \tilde{\chi}_{1}^{0} \tilde{\chi}_{1}^{0}}=\frac{m_{Z}}{\sqrt{2} v}\left(N_{13}^{2}-N_{14}^{2}\right) \sim 0.2 \times \frac{m_{Z}}{\sqrt{2} v}
$$

In the last step of the equation, we have used the information of $N_{13}$ and $N_{14}$ presented in figure 3. Moreover, it is interesting to see that although the benchmark point P4 in table 3 is hard to be excluded by sparticle direct search experiments at the LHC, its SD cross section is quite large and so the point will be tested by future dark matter direct search experiments.

In getting figure 5, we use the package micrOMEGAs [74] to calculate the cross sections. We choose its default setting $\sigma_{\pi N}=34 \mathrm{MeV}$ and $\sigma_{0}=42 \mathrm{MeV}$ as input. We checked that if we take $\sigma_{\pi N}=59 \mathrm{MeV}$ from [75] and $\sigma_{0}=58 \mathrm{MeV}$ from [76-78], the SI cross section will be enhanced by a factor from $20 \%$ to $40 \%$, and this does not affect the conclusions presented in this work.

\section{Conclusions}

With the great discovery of the $125 \mathrm{GeV}$ Higgs boson and the increased bounds on sparticle masses at LHC Run I, the NS scenario in MSSM has become theoretically unsatisfactory. By contrast, the situation may be improved greatly in the NMSSM. This motivates us to scrutinize the impact of the direct searches for SUSY at LHC Run I on the naturalness of the NMSSM. 
We start our study by scanning the vast parameter space of the NMSSM to get the region where the fine tuning measures $\Delta_{Z}$ and $\Delta_{h}$ at electroweak scale are less than about 50. In this process, we considered various experimental constraints such as DM relic density, LUX limits on the scattering rate of DM with nucleon and the $125 \mathrm{GeV}$ Higgs data on the model. We classify the surviving samples into four types: for Type I samples, $h_{1}$ corresponds to the SM-like Higgs boson and $\tilde{\chi}_{1}^{0}$ is Bino-dominated, while for Type II, III and IV samples, $h_{2}$ acts as the SM-like Higgs boson with $\tilde{\chi}_{1}^{0}$ being Bino-, Singlino- and Higgsino-dominated respectively. After these preparations, we specially study the influence of the direct searches for SUSY on the samples. We implement the direct search constraints by the packages FastLim and SModelS and also by simulating the electroweakino and stop production processes. Our results indicate that although the direct search experiments are effective in excluding the samples, the parameter intervals for the region and also the minimum reaches of $\Delta_{Z}$ and $\Delta_{h}$ are scarcely changed by the constraints, which implies that, contrary to general belief, the fine tuning of the NMSSM does not get worse after LHC Run I. Our results also indicate that the lowest fine-tuning comes from type III and type IV samples, for which $\Delta_{Z}$ and $\Delta_{h}$ may be as low as about 2 without conflicting with any experimental constraints.

Considering that the type IV samples were scarcely studied in previous literatures and that they have similar phenomenology to that of the NS scenario in the MSSM, we investigate the essential features of this kind of samples. We find that they are characterized by strong mixings between Higgsinos and Singlino in forming mass eigenstates called neutralinos. As a result, the lightest neutralino $\tilde{\chi}_{1}^{0}$ as the DM candidate has significant Singlino component so that it can easily reach the measured DM relic density, and meanwhile the mass splittings among the Higgsino-dominated particles $\tilde{\chi}_{1}^{0}, \tilde{\chi}_{2}^{0}$ and $\tilde{\chi}_{1}^{ \pm}$are usually larger than $30 \mathrm{GeV}$. These features make the samples rather special. For example, we show that due to the rich decay products of the lighter scalar top quark, its lower mass bound is decreased by about $100 \mathrm{GeV}$ in comparison with that in the NS scenario of the MSSM, and that the neutralino-chargino sector of the samples can be readily tested either through searching for $3 l+E_{T}^{\text {miss }}$ signal at $14 \mathrm{TeV}$ LHC or through future dark matter direct detection experiments.

In summary, we conclude that so far the fine tuning of the NMSSM is scarcely affected by the direct searches for SUSY at LHC Run I, and it can still predict $Z$ boson mass and the SM-like Higgs boson mass in a natural way. This conclusion, however, may be altered by the upcoming $14 \mathrm{TeV}$ LHC experiments and DM matter direct search experiments.

\section{Acknowledgments}

This work was supported by the National Natural Science Foundation of China (NNSFC) under grant No. 11575053, 11275245 and 11305050. 


\begin{tabular}{|c|c|c|c|c|}
\hline Name & Description & $\sqrt{s}$ & $\mathcal{L}_{\text {int }}\left(\mathrm{fb}^{-1}\right)$ & Ref. \\
\hline ATLAS-CONF-2013-062 & 1-2 leptons $+3-6$ jets $+E_{T}^{\text {miss }}$ (squarks and gluino) & \multirow{8}{*}{$8 \mathrm{TeV}$} & 20.3 & {$[79]$} \\
\hline ATLAS-CONF-2013-061 & 0 -1 lepton $+\geq 3$ b-jets $+E_{T}^{\text {miss }}$ (3rd gen. squarks) & & 20.1 & {$[80]$} \\
\hline ATLAS-CONF-2013-054 & 0 lepton $+\geq 7-10$ jets $+E_{T}^{\text {miss }}$ (squarks and gluino) & & 20.3 & {$[81]$} \\
\hline ATLAS-CONF-2013-053 & 0 lepton +2 b-jets $+E_{T}^{\text {miss }}$ (sbottom and stop) & & 20.1 & {$[82]$} \\
\hline ATLAS-CONF-2013-048 & 2 leptons $(+$ jets $)+E_{T}^{\text {miss }}$ (stop) & & 20.3 & [83] \\
\hline ATLAS-CONF-2013-047 & 0 lepton $+2-6$ jets $+E_{T}^{\text {miss }}$ (squarks and gluino) & & 20.3 & {$[84]$} \\
\hline ATLAS-CONF-2013-037 & 1 lepton $+4(1 \mathrm{~b}-)$ jets $+E_{T}^{\text {miss }}$ (stop) & & 20.7 & [85] \\
\hline ATLAS-CONF-2013-024 & 0 lepton $+\left(2\right.$ b-)jets $+E_{T}^{\text {miss }}$ (stop $)$ & & 20.5 & {$[86]$} \\
\hline
\end{tabular}

Table 6. Experiments in the Fastlim database.

\section{A Fastlim and SModelS}

In this section, we briefly introduce the packages Fastlim [66] and SModelS [67], which can be used to implement the constraints from the direct searches for sparticles at LHC Run I in an easy and fast way.

\section{A.1 Fastlim}

Fastlim is a package that limits SUSY parameter space using the LHC-8 TeV data. It incorporates in its database cut efficiency tables for different sparticle production processes in simplified model framework. Now it supports the processes of gluino pair production and third generation squark pair productions, and it involves many decay modes of gluino and the squarks, such as $\tilde{g} \rightarrow \tilde{t}_{1,2}^{*} t \rightarrow \tilde{\chi}_{1}^{0} t \bar{t}$ or $\tilde{g} \rightarrow q \bar{q} \tilde{\chi}_{1}^{0}$ and $\tilde{b}_{1,2} \rightarrow b \tilde{\chi}_{1}^{0}$ where $q$ stands for the first two generation quarks. Any processes that contain the same final states at the detector level are combined by Fastlim to improve the signal significance.

In this work, we only use the experiments of searching for gluino and squarks, which are listed in table 6. This is because that Fastlim gives better limits for strong SUSY productions in comparison with the package SModelS [67].

\section{A.2 SModelS}

SModelS have the same function as that of Fastlim, but it could give better limits for slepton productions and electroweakino productions. SModelS has implemented the information about following experiments:

- Direct slepton searches (ATLAS): ATLAS-CONF-2013-049 [87].

- Direct slepton searches (CMS): SUS-12-022 [88], SUS-13-006 [89].

- Electroweakino searches (ATLAS): ATLAS-CONF-2013-028 [90], ATLAS-CONF2013-035 [91], ATLAS-CONF-2013-036 [92], ATLAS-CONF-2013-093 [93].

- Electroweakino searches (CMS): SUS-12-022 [88], SUS-13-006 [89], SUS-13-017 [94],

and it contains the cross section limits at $95 \%$ confidence level for above analyses. If one parameter point predicts cross sections larger than those presented in the analyses, it will be excluded. Otherwise, it is allowed. 
Note that the efficiencies or the upper bounds on SUSY signals in the database of the two packages are usually based on certain assumptions, which may not be applied to some parameter points encountered in our scan. In this case, the encountered point is considered to be experimentally allowed. Also note that the packages are based on the preliminary analyses of the ATLAS and CMS groups, which were done in 2013. Given that most of these analyses have been updated in past two years, a more powerful exclusion capability may be obtained if one repeats the updated analyses by detailed Monte Carlo simulation. Anyhow, these two packages can serve as useful tools to exclude some SUSY parameter points.

\section{B Details of our simulation}

In our study, any point that passed Fastlim and SModelS is further tested by simulations to see whether it survives the constraints from the direct search experiments. In detail, we first use MadGraph/MadEvent [95, 96] to generate parton level events for certain sparticle production processes, and feed them into Pythia [97] for parton showering and hadronization. Then we use the package CheckMATE [99] where a well-tuned Delphes [98] is provided for the detector simulation and analyses. We define $R \equiv \max \left\{S_{i} / S_{i \text { obs }}^{95}\right\}$ to decide whether the point survives the analysis, where $S_{i}$ stands for the simulated signal events in the $i$ th signal region of the analysis, and $S_{i, \mathrm{obs}}^{95}$ represents the $95 \%$ C.L. upper limit of the event number in the signal region. If $R>1$, the parameter point is excluded by the analysis and otherwise it is allowed.

Since the fine tuning of the NMSSM at the electroweak scale is mainly affected by its chargino-neutralino sector and stop sector, we repeat by simulation the ATLAS analyses in $[71,100,102]$ and [104]. All these analyses are based on $20.3 \mathrm{fb}^{-1}$ data at the LHC- $8 \mathrm{TeV}$ with the former two presenting so far the strictest limits on electroweakino productions, and the latter two providing the tightest constraints on the pair productions of third generation squarks. In the following, we briefly introduce these analyses.

\section{B.1 Search for electroweakino at the LHC}

The analysis [100] targets final states with two leptons and large $E_{T}^{\text {miss }}$. In our simulation of the analysis, we mainly focus on the signal region named "SR-Zjets", which is specifically designed for the process $p p \rightarrow \tilde{\chi}_{2}^{0} \tilde{\chi}_{1}^{ \pm} \rightarrow Z \tilde{\chi}_{1}^{0} W \tilde{\chi}_{1}^{0} \rightarrow \ell \ell \tilde{\chi}_{1}^{0} q q \tilde{\chi}_{1}^{0}$. This signal region requires that the two leading leptons should be same flavor but opposite sign (SFOS), and that their invariant mass locates at $Z$-peak. As was pointed out in [100], it provides the strongest constraints on the chargino-neutralino sector among the anlayses with dilepton final state.

The analysis in [71] also searches for electroweakino productions but with final states of three leptons and large $E_{T}^{\text {miss }}$. Here we concentrate on the signal region named "SR0 $\tau$ a" which is optimized for processes $p p \rightarrow \tilde{\chi}_{2}^{0} \tilde{\chi}_{1}^{ \pm} \rightarrow Z^{(*)} \tilde{\chi}_{1}^{0} W^{(*)} \tilde{\chi}_{1}^{0} \rightarrow \ell \ell \tilde{\chi}_{1}^{0} \ell \nu \tilde{\chi}_{1}^{0}$. This region requires a pair of SFOS leptons in its signal, and utilizes the transverse mass $m_{T}=$ $\sqrt{2\left|\vec{p}_{T}^{\ell}\right|\left|\vec{E}_{T}^{\text {miss }}\right|-2 \vec{p}_{T}^{\ell} \cdot \vec{E}_{T}^{\text {miss }}}$ (here $p_{T}^{l}$ is the transverse momentum of the lepton not forming the SFOS lepton pair) to suppress the SM background. It considers twenty bins, which are 
categorized by the SFOS leptons' invariant mass, $m_{T}$ and $E_{T}^{\text {miss }}$, to maximize its sensitivity to different mass spectrums of $\tilde{\chi}_{2}, \tilde{\chi}_{1}^{ \pm}$and $\tilde{\chi}_{1}^{0}$.

We remind that in the NS scenarios of the NMSSM, all $\tilde{\chi}_{i}^{0} \tilde{\chi}_{1}^{ \pm}$associated production processes with $i=2,3,4,5$ may contribute sizably to the trilepton signal, so in our simulation we include all these contributions. By contrast, SModelS does not combine processes that have the same final states at the detector level. Moreover, in our analysis we combine the signal region "SR-Zjets" with the bins in "SR0 $\tau$ a" to maximize the discovery significance by the $\mathrm{CL}_{s}$ method in RooStats as we did in [9].

\section{B.2 Search for stops at the LHC}

In the NS scenario of the NMSSM, $\tilde{t}_{1}$ usually decays like $\tilde{t}_{1} \rightarrow \tilde{\chi}_{2,3}^{0} t \rightarrow \tilde{\chi}_{1}^{0} Z^{(*)} t$ or $\tilde{t}_{1} \rightarrow$ $\tilde{\chi}_{1}^{+} b \rightarrow \tilde{\chi}_{1}^{0} W^{(*)} b$. Considering that these two-step topologies haven't been included in the database of Fastlim, we repeat recent ATLAS analyses on stop pair productions, which were presented in [102] and [104].

The analysis in [102] searches for stops in final states containing exactly one isolated lepton, at least two jets and a large $E_{T}^{\text {miss }}$. It contains fifteen signal regions targeting a large number of stop pair production scenarios, where stop may decay like $\tilde{t}_{1} \rightarrow t \tilde{\chi}_{1}^{0}$, $\tilde{t}_{1} \rightarrow b W^{(*)} \tilde{\chi}_{1}^{0}$ and $\tilde{t}_{1} \rightarrow b \tilde{\chi}_{1}^{ \pm} \rightarrow b W^{(*)} \tilde{\chi}_{1}^{0}$. Especially, nine of these signal regions are designed for the latter decay with different mass relations among $\tilde{t}_{1}, \tilde{\chi}_{1}^{ \pm}$and $\tilde{\chi}_{1}^{0}$, which determines the kinematic properties of the process. These nine regions are particularly relevant to our study. In general, the signal regions are discriminated by different kinematic cuts on the leptons and (b-)jets, $E_{T}^{\text {miss }}, m_{T}$, b-jet multiplicity and the asymmetric transverse mass $a m_{T 2}$ [103].

The analysis in [104] targets the process $p p \rightarrow \tilde{t}_{2}^{*} \tilde{t}_{2}$ with $\tilde{t}_{2} \rightarrow \tilde{t}_{1} Z \rightarrow \tilde{\chi}_{1}^{0} Z t$, and it searches for the signal that contains a SFOS pair of leptons with their invariant mass near $m_{Z}$, at least one b-jet and a large $E_{T}^{\text {miss }}$. Obviously, if $\tilde{t}_{2}$ is significantly heavier than $\tilde{t}_{1}$, the $Z$ boson is highly boosted. As a result, the transverse momentum of the dilepton system $p_{T}(\ell \ell)$ tends to be high and the azimuthal separation $\Delta \phi(\ell \ell)$ prefers to be low. On the other side, if the mass splitting between $\tilde{t}_{1}$ and $\tilde{\chi}_{1}^{0}$ is large, high jet multiplicity is expected. These facts motivates physicists to define five signal regions, which are called "SR2(A,B,C)" and "SR3(A,B)" respectively, by the number of leptons in the signal, the jet multiplicity and whether the $\mathrm{Z}$ boson is boosted. For example, the signal regions "SR2(A,B,C)" require that the signal events contain exactly two signal leptons and the $Z$ boson is boosted. The regions "SR2A" and "SR2B" are optimized for low jet multiplicity, while the region "SR2C" is designed for high jet multiplicity case. 
Open Access. This article is distributed under the terms of the Creative Commons Attribution License (CC-BY 4.0), which permits any use, distribution and reproduction in any medium, provided the original author(s) and source are credited.

\section{References}

[1] H.E. Haber and G.L. Kane, The search for supersymmetry: probing physics beyond the standard model, Phys. Rept. 117 (1985) 75 [INSPIRE].

[2] A. Djouadi, The anatomy of electro-weak symmetry breaking. II. The Higgs bosons in the minimal supersymmetric model, Phys. Rept. 459 (2008) 1 [hep-ph/0503173] [INSPIRE].

[3] U. Ellwanger, C. Hugonie and A.M. Teixeira, The next-to-minimal supersymmetric standard model, Phys. Rept. 496 (2010) 1 [arXiv:0910.1785] [INSPIRE].

[4] M. Maniatis, The next-to-minimal supersymmetric extension of the standard model reviewed, Int. J. Mod. Phys. A 25 (2010) 3505 [arXiv:0906. 0777] [InSPIRE].

[5] H. Baer, V. Barger, P. Huang and X. Tata, Natural supersymmetry: LHC, dark matter and ILC searches, JHEP 05 (2012) 109 [arXiv:1203.5539] [INSPIRE].

[6] H. Baer et al., Radiative natural supersymmetry: reconciling electroweak fine-tuning and the Higgs boson mass, Phys. Rev. D 87 (2013) 115028 [arXiv:1212.2655] [InSPIRE].

[7] ATLAS collaboration, Search for squarks and gluinos with the ATLAS detector in final states with jets and missing transverse momentum and $20.3 \mathrm{fb}^{-1}$ of $\sqrt{\mathrm{s}}=8 \mathrm{TeV}$ proton-proton collision data, ATLAS-CONF-2013-047 (2013).

[8] CMS collaboration, Search for new physics in the multijet and missing transverse momentum final state in proton-proton collisions at $\sqrt{s}=8 \mathrm{TeV}$, JHEP 06 (2014) 055 [arXiv: 1402.4770] [INSPIRE].

[9] J. Cao, Y. He, L. Shang, W. Su and Y. Zhang, Testing the light dark matter scenario of the MSSM at the LHC, JHEP 03 (2016) 207 [arXiv:1511.05386] [INSPIRE].

[10] ATLAS collaboration, Observation of a new particle in the search for the standard model Higgs boson with the ATLAS detector at the LHC, Phys. Lett. B 716 (2012) 1 [arXiv:1207.7214] [INSPIRE].

[11] CMS collaboration, Observation of a new boson at a mass of $125 \mathrm{GeV}$ with the CMS experiment at the LHC, Phys. Lett. B 716 (2012) 30 [arXiv:1207.7235] [INSPIRE].

[12] H. Baer, V. Barger and A. Mustafayev, Implications of a $125 \mathrm{GeV}$ Higgs scalar for LHC SUSY and neutralino dark matter searches, Phys. Rev. D 85 (2012) 075010 [arXiv:1112.3017] [INSPIRE].

[13] S. Heinemeyer, O. Stål and G. Weiglein, Interpreting the LHC Higgs search results in the MSSM, Phys. Lett. B 710 (2012) 201 [arXiv:1112.3026] [INSPIRE].

[14] A. Arbey, M. Battaglia, A. Djouadi, F. Mahmoudi and J. Quevillon, Implications of a $125 \mathrm{GeV}$ Higgs for supersymmetric models, Phys. Lett. B 708 (2012) 162 [arXiv:1112.3028] [INSPIRE].

[15] P. Draper, P. Meade, M. Reece and D. Shih, Implications of a $125 \mathrm{GeV}$ Higgs for the MSSM and low-scale SUSY breaking, Phys. Rev. D 85 (2012) 095007 [arXiv:1112.3068] [inSPIRE].

[16] M. Carena, S. Gori, N.R. Shah and C.E.M. Wagner, A $125 \mathrm{GeV}$ SM-like Higgs in the MSSM and the $\gamma \gamma$ rate, JHEP 03 (2012) 014 [arXiv:1112.3336] [INSPIRE]. 
[17] J. Cao, Z. Heng, D. Li and J.M. Yang, Current experimental constraints on the lightest Higgs boson mass in the constrained MSSM, Phys. Lett. B 710 (2012) 665 [arXiv:1112.4391] [INSPIRE].

[18] J. Cao, L. Shang, P. Wu, J.M. Yang and Y. Zhang, Interpreting the galactic center gamma-ray excess in the NMSSM, JHEP 10 (2015) 030 [arXiv:1506.06471] [INSPIRE].

[19] J. Cao, L. Shang, P. Wu, J.M. Yang and Y. Zhang, Supersymmetry explanation of the Fermi galactic center excess and its test at LHC run II, Phys. Rev. D 91 (2015) 055005 [arXiv:1410.3239] [INSPIRE].

[20] J. Guo, J. Li, T. Li and A.G. Williams, NMSSM explanations of the galactic center gamma ray excess and promising LHC searches, Phys. Rev. D 91 (2015) 095003 [arXiv: 1409.7864] [INSPIRE].

[21] X.-J. Bi, L. Bian, W. Huang, J. Shu and P.-F. Yin, Interpretation of the galactic center excess and electroweak phase transition in the NMSSM, Phys. Rev. D 92 (2015) 023507 [arXiv: 1503.03749] [INSPIRE].

[22] U. Ellwanger, A Higgs boson near $125 \mathrm{GeV}$ with enhanced di-photon signal in the NMSSM, JHEP 03 (2012) 044 [arXiv:1112.3548] [INSPIRE].

[23] Z. Kang, J. Li and T. Li, On naturalness of the MSSM and NMSSM, JHEP 11 (2012) 024 [arXiv:1201.5305] [INSPIRE].

[24] S.F. King, M. Mühlleitner and R. Nevzorov, NMSSM Higgs benchmarks near $125 \mathrm{GeV}$, Nucl. Phys. B 860 (2012) 207 [arXiv:1201.2671] [INSPIRE].

[25] J.-J. Cao, Z.-X. Heng, J.M. Yang, Y.-M. Zhang and J.-Y. Zhu, A SM-like Higgs near $125 \mathrm{GeV}$ in low energy SUSY: a comparative study for MSSM and NMSSM, JHEP 03 (2012) 086 [arXiv:1202.5821] [InSPIRE].

[26] K. Choi, S.H. Im, K.S. Jeong and M. Yamaguchi, Higgs mixing and diphoton rate enhancement in NMSSM models, JHEP 02 (2013) 090 [arXiv:1211.0875] [INSPIRE].

[27] S.F. King, M. Mühlleitner, R. Nevzorov and K. Walz, Natural NMSSM Higgs bosons, Nucl. Phys. B 870 (2013) 323 [arXiv: 1211.5074] [INSPIRE].

[28] M. Badziak, M. Olechowski and S. Pokorski, New regions in the NMSSM with a $125 \mathrm{GeV}$ Higgs, JHEP 06 (2013) 043 [arXiv: 1304.5437] [INSPIRE].

[29] S. Moretti, S. Munir and P. Poulose, 125 GeV Higgs boson signal within the complex NMSSM, Phys. Rev. D 89 (2014) 015022 [arXiv:1305.0166] [INSPIRE].

[30] D. Das, U. Ellwanger and A.M. Teixeira, Modified signals for supersymmetry in the NMSSM with a singlino-like LSP, JHEP 04 (2012) 067 [arXiv:1202.5244] [INSPIRE].

[31] D. Albornoz Vasquez et al., The 125 GeV Higgs in the NMSSM in light of LHC results and astrophysics constraints, Phys. Rev. D 86 (2012) 035023 [arXiv:1203.3446] [INSPIRE].

[32] U. Ellwanger, Testing the Higgsino-singlino sector of the NMSSM with trileptons at the LHC, JHEP 11 (2013) 108 [arXiv:1309.1665] [INSPIRE].

[33] J.S. Kim and T.S. Ray, The Higgsino-singlino world at the Large Hadron Collider, Eur. Phys. J. C 75 (2015) 40 [arXiv:1405.3700] [INSPIRE].

[34] U. Ellwanger and C. Hugonie, The semi-constrained NMSSM satisfying bounds from the LHC, LUX and Planck, JHEP 08 (2014) 046 [arXiv:1405.6647] [INSPIRE]. 
[35] U. Ellwanger and A.M. Teixeira, NMSSM with a singlino LSP: possible challenges for searches for supersymmetry at the LHC, JHEP 10 (2014) 113 [arXiv:1406.7221] [INSPIRE].

[36] B. Dutta, Y. Gao and B. Shakya, Light Higgsino decays as a probe of the NMSSM, Phys. Rev. D 91 (2015) 035016 [arXiv:1412.2774] [INSPIRE].

[37] B. Allanach, M. Badziak, C. Hugonie and R. Ziegler, Light sparticles from a light singlet in gauge mediation, Phys. Rev. D 92 (2015) 015006 [arXiv: 1502.05836] [INSPIRE].

[38] A. Chakraborty, D.K. Ghosh, S. Mondal, S. Poddar and D. Sengupta, Probing the NMSSM via Higgs boson signatures from stop cascade decays at the LHC, Phys. Rev. D 91 (2015) 115018 [arXiv:1503.07592] [INSPIRE].

[39] J. Beuria, A. Chatterjee, A. Datta and S.K. Rai, Two light stops in the NMSSM and the LHC, JHEP 09 (2015) 073 [arXiv: 1505.00604] [inSPIRE].

[40] C.T. Potter, Natural NMSSM with a light singlet Higgs and singlino LSP, Eur. Phys. J. C 76 (2016) 44 [arXiv:1505.05554] [InSPIRE].

[41] R. Enberg, S. Munir, C. Pérez de los Heros and D. Werder, Prospects for Higgsino-singlino dark matter detection at IceCube and PINGU, arXiv:1506.05714 [INSPIRE].

[42] D. Barducci, G. Bélanger, C. Hugonie and A. Pukhov, Status and prospects of the NMSSM after LHC run-1, JHEP 01 (2016) 050 [arXiv: 1510.00246] [INSPIRE].

[43] J.S. Kim, D. Schmeier and J. Tattersall, Role of the ' $N$ ' in the natural NMSSM for the LHC, Phys. Rev. D 93 (2016) 055018 [arXiv:1510.04871] [InSPIRE].

[44] M. Badziak, M. Olechowski and P. Szczerbiak, Blind spots for neutralino dark matter in the NMSSM, JHEP 03 (2016) 179 [arXiv:1512.02472] [INSPIRE].

[45] J. Beuria, A. Chatterjee and A. Datta, Sbottoms of natural NMSSM at the LHC, arXiv: 1603.08463 [INSPIRE].

[46] Q.-F. Xiang, X.-J. Bi, P.-F. Yin and Z.-H. Yu, Searching for singlino-Higgsino dark matter in the NMSSM, arXiv:1606.02149 [INSPIRE].

[47] Planck collaboration, P.A.R. Ade et al., Planck 2013 results. XVI. Cosmological parameters, Astron. Astrophys. 571 (2014) A16 [arXiv: 1303.5076] [INSPIRE].

[48] WMAP collaboration, G. Hinshaw et al., Nine-year Wilkinson Microwave Anisotropy Probe (WMAP) observations: cosmological parameter results, Astrophys. J. Suppl. 208 (2013) 19 [arXiv:1212.5226] [INSPIRE].

[49] LUX collaboration, D.S. Akerib et al., Improved limits on scattering of weakly interacting massive particles from reanalysis of 2013 LUX data, Phys. Rev. Lett. 116 (2016) 161301 [arXiv: 1512.03506] [INSPIRE].

[50] LUX collaboration, D.S. Akerib et al., Results on the spin-dependent scattering of weakly interacting massive particles on nucleons from the run 3 data of the LUX experiment, Phys. Rev. Lett. 116 (2016) 161302 [arXiv:1602.03489] [INSPIRE].

[51] H. Baer, V. Barger, M. Savoy and X. Tata, Multi-channel assault on natural supersymmetry at the high luminosity LHC, arXiv: 1604.07438 [INSPIRE].

[52] U. Ellwanger and C. Hugonie, Higgs bosons near $125 \mathrm{GeV}$ in the NMSSM with constraints at the GUT scale, Adv. High Energy Phys. 2012 (2012) 625389 [arXiv:1203.5048] [INSPIRE].

[53] T. Cheng, J. Li, T. Li and Q.-S. Yan, Natural NMSSM confronting with the LHC7-8, Phys. Rev. D 89 (2014) 015015 [arXiv: 1304.3182] [INSPIRE]. 
[54] H. Baer, V. Barger and D. Mickelson, How conventional measures overestimate electroweak fine-tuning in supersymmetric theory, Phys. Rev. D 88 (2013) 095013 [arXiv:1309.2984] [INSPIRE].

[55] U. Ellwanger, G. Espitalier-Noël and C. Hugonie, Naturalness and fine tuning in the NMSSM: implications of early LHC results, JHEP 09 (2011) 105 [arXiv:1107.2472] [INSPIRE].

[56] M. Farina, M. Perelstein and B. Shakya, Higgs couplings and naturalness in $\lambda$-SUSY, JHEP 04 (2014) 108 [arXiv:1310.0459] [InSPIRE].

[57] U. Ellwanger and C. Hugonie, NMHDECAY 2.0: an updated program for sparticle masses, Higgs masses, couplings and decay widths in the NMSSM,

Comput. Phys. Commun. 175 (2006) 290 [hep-ph/0508022] [INSPIRE].

[58] U. Ellwanger, J.F. Gunion and C. Hugonie, NMHDECAY: a Fortran code for the Higgs masses, couplings and decay widths in the NMSSM, JHEP 02 (2005) 066 [hep-ph/0406215] [INSPIRE].

[59] P. Bechtle, S. Heinemeyer, O. Stål, T. Stefaniak and G. Weiglein, HiggsSignals: confronting arbitrary Higgs sectors with measurements at the Tevatron and the LHC,

Eur. Phys. J. C 74 (2014) 2711 [arXiv:1305.1933] [inSPIRE].

[60] P. Bechtle, S. Heinemeyer, O. Stål, T. Stefaniak and G. Weiglein, Probing the standard model with Higgs signal rates from the Tevatron, the LHC and a future ILC, JHEP 11 (2014) 039 [arXiv:1403.1582] [InSPIRE].

[61] O. Stål and T. Stefaniak, Constraining extended Higgs sectors with HiggsSignals, PoS (EPS-HEP 2013) 314 [arXiv: 1310.4039] [INSPIRE].

[62] M.D. Goodsell, K. Nickel and F. Staub, Two-loop corrections to the Higgs masses in the NMSSM, Phys. Rev. D 91 (2015) 035021 [arXiv:1411.4665] [INSPIRE].

[63] F. Staub et al., Higgs mass predictions of public NMSSM spectrum generators, Comput. Phys. Commun. 202 (2016) 113 [arXiv:1507.05093] [INSPIRE].

[64] P. Bechtle, O. Brein, S. Heinemeyer, G. Weiglein and K.E. Williams, HiggsBounds: confronting arbitrary Higgs sectors with exclusion bounds from LEP and the Tevatron, Comput. Phys. Commun. 181 (2010) 138 [arXiv:0811.4169] [InSPIRE].

[65] P. Bechtle, O. Brein, S. Heinemeyer, G. Weiglein and K.E. Williams, HiggsBounds 2.0.0: confronting neutral and charged Higgs sector predictions with exclusion bounds from LEP and the Tevatron, Comput. Phys. Commun. 182 (2011) 2605 [arXiv:1102.1898] [InSPIRE].

[66] M. Papucci, K. Sakurai, A. Weiler and L. Zeune, Fastlim: a fast LHC limit calculator, Eur. Phys. J. C 74 (2014) 3163 [arXiv: 1402.0492] [InSPIRE].

[67] S. Kraml et al., SModelS: a tool for interpreting simplified-model results from the LHC and its application to supersymmetry, Eur. Phys. J. C 74 (2014) 2868 [arXiv:1312.4175] [INSPIRE].

[68] A. Kobakhidze, N. Liu, L. Wu, J.M. Yang and M. Zhang, Closing up a light stop window in natural SUSY at LHC, Phys. Lett. B 755 (2016) 76 [arXiv:1511.02371] [INSPIRE].

[69] J. Cao, C. Han, L. Wu, J.M. Yang and Y. Zhang, Probing natural SUSY from stop pair production at the LHC, JHEP 11 (2012) 039 [arXiv:1206.3865] [INSPIRE]. 
[70] C. Han, K.-i. Hikasa, L. Wu, J.M. Yang and Y. Zhang, Current experimental bounds on stop mass in natural SUSY, JHEP 10 (2013) 216 [arXiv:1308.5307] [INSPIRE].

[71] ATLAS collaboration, Search for direct production of charginos and neutralinos in events with three leptons and missing transverse momentum in $\sqrt{s}=8 \mathrm{TeV}$ pp collisions with the ATLAS detector, JHEP 04 (2014) 169 [arXiv: 1402.7029] [INSPIRE].

[72] H. Baer and X. Tata, Multi-lepton signals from $W^{ \pm}$and $Z^{0}$ decays to gauginos at $\bar{p} p$ colliders, Phys. Lett. B 155 (1985) 278 [inSPIRE].

[73] P. Cushman et al., Snowmass CF1 summary: WIMP dark matter direct detection, arXiv: 1310.8327 [INSPIRE].

[74] G. Bélanger et al., Indirect search for dark matter with MicrOMEGAs_2.4, Comput. Phys. Commun. 182 (2011) 842 [arXiv:1004.1092] [InSPIRE].

[75] J.M. Alarcón, J. Martin Camalich and J.A. Oller, The chiral representation of the $\pi N$ scattering amplitude and the pion-nucleon sigma term, Phys. Rev. D 85 (2012) 051503 [arXiv:1110.3797] [INSPIRE].

[76] J.M. Alarcón, L.S. Geng, J. Martin Camalich and J.A. Oller, The strangeness content of the nucleon from effective field theory and phenomenology, Phys. Lett. B 730 (2014) 342 [arXiv:1209.2870] [INSPIRE].

[77] X.L. Ren, L.S. Geng, J. Martin Camalich, J. Meng and H. Toki, Octet baryon masses in next-to-next-to-next-to-leading order covariant baryon chiral perturbation theory, JHEP 12 (2012) 073 [arXiv:1209.3641] [INSPIRE].

[78] X.-L. Ren, L.-S. Geng and J. Meng, Scalar strangeness content of the nucleon and baryon sigma terms, Phys. Rev. D 91 (2015) 051502 [arXiv:1404.4799] [INSPIRE].

[79] ATLAS collaboration, Search for squarks and gluinos in events with isolated leptons, jets and missing transverse momentum at $\sqrt{s}=8 \mathrm{TeV}$ with the ATLAS detector, ATLAS-CONF-2013-062 (2013).

[80] ATLAS collaboration, Search for strong production of supersymmetric particles in final states with missing transverse momentum and at least three b-jets using $20.1 \mathrm{fb}^{-1}$ of $\mathrm{pp}$ collisions at $\sqrt{s}=8 \mathrm{TeV}$ with the ATLAS detector, ATLAS-CONF-2013-061 (2013).

[81] ATLAS collaboration, Search for new phenomena using final states with large jet multiplicities and missing transverse momentum with ATLAS in $20 \mathrm{fb}^{-1}$ of $\sqrt{\mathrm{s}}=8 \mathrm{TeV}$ proton-proton collisions, ATLAS-CONF-2013-054 (2013).

[82] ATLAS collaboration, Search for direct third generation squark pair production in final states with missing transverse momentum and two b-jets in $\sqrt{s}=8 \mathrm{TeV}$ pp collisions with the ATLAS detector, ATLAS-CONF-2013-053 (2013).

[83] ATLAS collaboration, Search for direct top squark pair production in final states with two leptons in $\sqrt{s}=8 \mathrm{TeV}$ pp collisions using $20 \mathrm{fb}^{-1}$ of ATLAS data, ATLAS-CONF-2013-048 (2013).

[84] ATLAS collaboration, Search for squarks and gluinos with the ATLAS detector in final states with jets and missing transverse momentum and $20.3 \mathrm{fb}^{-1}$ of $\sqrt{\mathrm{s}}=8 \mathrm{TeV}$ proton-proton collision data, ATLAS-CONF-2013-047 (2013).

[85] ATLAS collaboration, Search for direct top squark pair production in final states with one isolated lepton, jets and missing transverse momentum in $\sqrt{s}=8 \mathrm{TeV}$ pp collisions using $21 \mathrm{fb}^{-1}$ of ATLAS data, ATLAS-CONF-2013-037 (2013). 
[86] ATLAS collaboration, Search for direct production of the top squark in the all-hadronic $t \bar{t}+E_{T}^{\text {miss }}$ final state in $21 \mathrm{fb}^{-1}$ of $p$-p collisions at $\sqrt{s}=8 \mathrm{TeV}$ with the ATLAS detector, ATLAS-CONF-2013-024 (2013).

[87] ATLAS collaboration, Search for direct-slepton and direct-chargino production in final states with two opposite-sign leptons, missing transverse momentum and no jets in $20 \mathrm{fb}^{-1}$ of pp collisions at $\sqrt{s}=8 \mathrm{TeV}$ with the ATLAS detector, ATLAS-CONF-2013-049 (2013).

[88] CMS collaboration, Search for electroweak production of charginos, neutralinos and sleptons using leptonic final states in pp collisions at $\sqrt{s}=8 \mathrm{TeV}$, CMS-PAS-SUS-12-022 (2013).

[89] CMS collaboration, Searches for electroweak production of charginos, neutralinos, and sleptons decaying to leptons and $W, Z$, and Higgs bosons in pp collisions at $8 \mathrm{TeV}$, Eur. Phys. J. C 74 (2014) 3036 [arXiv: 1405.7570] [CMS-SUS-13-006] [INSPIRE].

[90] ATLAS collaboration, Search for electroweak production of supersymmetric particles in final states with at least two hadronically decaying taus and missing transverse momentum with the ATLAS detector in proton-proton collisions at $\sqrt{s}=8 \mathrm{TeV}$,

ATLAS-CONF-2013-028 (2013).

[91] ATLAS collaboration, Search for direct production of charginos and neutralinos in events with three leptons and missing transverse momentum in $21 \mathrm{fb}^{-1}$ of pp collisions at $\sqrt{s}=8 \mathrm{TeV}$ with the ATLAS detector, ATLAS-CONF-2013-035 (2013).

[92] ATLAS collaboration, Search for supersymmetry in events with four or more leptons in $21 \mathrm{fb}^{-1}$ of $\mathrm{pp}$ collisions at $\sqrt{\mathrm{s}}=8 \mathrm{TeV}$ with the ATLAS detector, ATLAS-CONF-2013-036 (2013).

[93] ATLAS collaboration, Search for chargino and neutralino production in final states with one lepton, two b-jets consistent with a Higgs boson and missing transverse momentum with the ATLAS detector in 20.3 fb $b^{-1}$ of $\sqrt{s}=8 \mathrm{TeV}$ pp collisions, ATLAS-CONF-2013-093 (2013).

[94] CMS collaboration, Search for electroweak production of charginos and neutralinos in final states with a Higgs boson in pp collisions at $\sqrt{s}=8 \mathrm{TeV}$, CMS-PAS-SUS-13-017 (2013).

[95] J. Alwall et al., The automated computation of tree-level and next-to-leading order differential cross sections and their matching to parton shower simulations, JHEP 07 (2014) 079 [arXiv:1405.0301] [InSPIRE].

[96] J. Alwall, M. Herquet, F. Maltoni, O. Mattelaer and T. Stelzer, MadGraph 5: going beyond, JHEP 06 (2011) 128 [arXiv:1106.0522] [InSPIRE].

[97] T. Sjöstrand, S. Mrenna and P.Z. Skands, PYTHIA 6.4 physics and manual, JHEP 05 (2006) 026 [hep-ph/0603175] [INSPIRE].

[98] DELPHES 3 collaboration, J. de Favereau et al., DELPHES 3: a modular framework for fast simulation of a generic collider experiment, JHEP 02 (2014) 057 [arXiv:1307.6346] [INSPIRE].

[99] M. Drees, H. Dreiner, D. Schmeier, J. Tattersall and J.S. Kim, CheckMATE: confronting your favourite new physics model with LHC data, Comput. Phys. Commun. 187 (2015) 227 [arXiv:1312.2591] [INSPIRE].

[100] ATLAS collaboration, Search for direct production of charginos, neutralinos and sleptons in final states with two leptons and missing transverse momentum in pp collisions at $\sqrt{s}=8 \mathrm{TeV}$ with the ATLAS detector, JHEP 05 (2014) 071 [arXiv:1403.5294] [INSPIRE]. 
[101] UA1 collaboration, G. Arnison et al., Experimental observation of isolated large transverse energy electrons with associated missing energy at $\sqrt{s}=540 \mathrm{GeV}$, Phys. Lett. B 122 (1983) 103 [INSPIRE].

[102] ATLAS collaboration, Search for top squark pair production in final states with one isolated lepton, jets and missing transverse momentum in $\sqrt{s}=8 \mathrm{TeV}$ pp collisions with the ATLAS detector, JHEP 11 (2014) 118 [arXiv:1407.0583] [INSPIRE].

[103] Y. Bai, H.-C. Cheng, J. Gallicchio and J. Gu, Stop the top background of the stop search, JHEP 07 (2012) 110 [arXiv:1203.4813] [InSPIRE].

[104] ATLAS collaboration, Search for direct top squark pair production in events with a $Z$ boson, b-jets and missing transverse momentum in $\sqrt{s}=8 \mathrm{TeV} p p$ collisions with the ATLAS detector, Eur. Phys. J. C 74 (2014) 2883 [arXiv:1403.5222] [INSPIRE]. 